\title{
Role of polynucleotide phosphorylase in sRNA function in Escherichia coli
}

\author{
NICHOLAS DE LAY and SUSAN GOTTESMAN
}

Laboratory of Molecular Biology, National Cancer Institute, Bethesda, Maryland 20892, USA

\begin{abstract}
In Escherichia coli, many small noncoding regulatory RNAs (sRNAs) post-transcriptionally regulate gene expression by basepairing to mRNAs in a process that is mediated by the RNA chaperone $\mathrm{Hfq}$. Binding of the sRNA to the mRNA can lead to increased or decreased mRNA stability and/or translation. It is not known if proteins other than Hfq are necessary for this process. In order to identify additional genes required for the post-transcriptional regulation of gene expression by $\mathrm{Hfq}$ dependent sRNAs, we developed a novel combined genetic selection and screen for mutants defective in sRNA regulation. In our combined genetic selection and screen, we isolated hfq mutants and mutants in pnp, encoding polynucleotide phosphorylase (PNPase). We show that loss-of-function mutations in pnp result in a decreased stability of several sRNAs including RyhB, SgrS, and CyaR and also decrease both the negative and positive regulation by sRNAs. The defect in stability of CyaR and in negative and positive regulation are suppressed by deletion mutations in RNase E. Altogether, our results suggest that the lack of sRNA-mediated regulation in the absence of an active form of PNPase is due to the rapid turnover of sRNA resulting from an increase in RNase E activity and/or an increase in access of other ribonucleases to sRNAs.
\end{abstract}

Keywords: Hfq; RNase E; polynucleotide phosphorylase

\section{INTRODUCTION}

The post-transcriptional regulation of gene expression by small noncoding RNAs is a critical process that allows bacteria to rapidly adapt to ever-changing internal and environmental conditions. In Escherichia coli, $\sim 100$ sRNAs that typically range in size from 50 to $250 \mathrm{nt}$ have been identified (Argaman et al. 2001; Wassarman et al. 2001; Chen et al. 2002; Vogel et al. 2003; Zhang et al. 2003). Approximately one-third of these sRNAs have been shown to bind to the RNA chaperone Hfq (Wassarman et al. 2001; Zhang et al. 2003), which is homologous to the eukaryotic family of Sm splicing proteins. Hfq was first identified in $E$. coli as a host factor required for the replication of the phage Q $\beta$ (Franze de Fernandez et al. 1972) and later assigned to the gene now called $h f q$ (Kajitani and Ishihama 1991). Its role in sRNA-mediated post-transcriptional regulation was discovered much later when $h f q$ mutants were found to be defective in rpoS translation in both Salmonella and E. coli (Brown and Elliott 1996; Muffler et al. 1996). Hfq catalyzes

Reprint requests to: Susan Gottesman, Laboratory of Molecular Biology, National Cancer Institute, Bethesda, MD 20892, USA; susang@ helix.nih.gov; fax: (301) 496-3875.

Article published online ahead of print. Article and publication date are at http://www.rnajournal.org/cgi/doi/10.1261/rna.2531211. the pairing of sRNAs to near-complementary sequences, typically in the $5^{\prime}$ untranslated region ( 5 ' UTR) of mRNAs (for reviews, see Valentin-Hansen et al. 2004; Brennan and Link 2007).

In some cases, binding of the sRNA to a sequence in the 5' UTR of the mRNA can stimulate translation of the mRNA. The best-studied example of this positive regulation is the post-transcriptional regulation of RpoS, the stationary phase sigma factor, by the sRNAs DsrA (Lease et al. 1998; Majdalani et al. 1998), RprA (Majdalani et al. 2001), and ArcZ (Mandin and Gottesman 2010). Basepairing between an upstream region of the $5^{\prime}$ UTR of the rpoS mRNA and a region encompassing the ribosome binding site normally prevents efficient translation of RpoS. However, binding of DsrA, RprA, or ArcZ to the upstream sequence increases accessibility of the Shine-Dalgarno sequence to ribosomes, resulting in an increased level of translation. Alternatively, binding of an sRNA to an mRNA can inhibit translation by binding to the translation initiation region of the mRNA and, in many cases, can lead to the coupled degradation of the sRNA and mRNA. For example, binding of RyhB sRNA to the translation initiation region of the $s o d B$ or $s d h C D A B$ mRNAs leads to an inhibition of translation and the coupled degradation of both RyhB and the targeted mRNAs (Massé et al. 2003). 
As mentioned above, the role of Hfq in sRNA regulation was deduced indirectly, as a result of studies of the phenotype of an $h f q$ mutant and the known RNA binding properties of the Hfq protein. While in vitro studies have demonstrated that Hfq alone can promote the association of sRNA and mRNA, no genetic search to determine whether other proteins are essential for in vivo regulation has been reported. We report here the development and application of a combined genetic selection and screen for mutants defective in the post-transcriptional regulation of gene expression by sRNAs. As expected, mutations in $h f q$ were identified. The only other mutations that came out of this search were mutations in pnp, encoding PNPase, a $3^{\prime}$ to $5^{\prime}$ exonuclease, providing insight to an unexpected role of this protein in bacterial sRNA regulation.

\section{RESULTS}

Hfq is necessary for both the stability of sRNAs and for sRNA-dependent regulation in E. coli (for review, see Waters and Storz 2008). We sought to identify other genes that are required for the post-transcriptional regulation of gene expression by these Hfq-dependent trans-encoded sRNAs. We developed a combined genetic selection and screen for mutants that are defective in the post-transcriptional regulation of two independent mRNAs, $s d h C D A B$ and cirA, by two different sRNAs, RyhB and OmrB, respectively. sdhCDAB encodes succinate dehydrogenase, which is essential for the ability of $E$. coli to grow on minimal medium containing succinate as the sole carbon source, and cirA encodes an outer membrane protein that binds an iron-chelating siderophore and some colicins.

The selection portion of this method is based on work of Massé and Gottesman, who found that a fur mutant constitutively expressed the sRNA RyhB, which in turn down-regulated the $s d h C D A B$ mRNA. As a consequence, the fur mutant was unable to grow on minimal medium containing succinate as a sole carbon source. Introduction of an $h f q$ deletion into the fur mutant restored growth on succinate minimal medium (Massé and Gottesman 2002). The genetic screen portion of this method is based on the demonstration that expression of the sRNA OmrB resulted in decreased expression of a $\operatorname{cir} A-l a c Z$ translation fusion, and that this effect was blocked in an $h f q^{-}$strain (Guillier and Gottesman 2008).

Our genetic selection/screen assumed that mutations in any gene essential for the post-transcriptional regulation of gene expression by sRNAs would result in a similar phenotype as introduction of an $h f q$ deletion. The strain used was MG1193/pBR-plac-OmrB, a fur ${ }^{-}$strain harboring a cirA-lac Z fusion and overexpressing OmrB from a $\mathrm{P}_{\text {lac }}$ promoter on a plasmid. It was unable to grow on succinate and formed white colonies on plates containing isopropyl $\beta$-D-1-thiogalactopyranoside (IPTG) and 5-bromo-4-chloro3-indolyl- $\beta$-D-galactopyranoside (X-Gal). An $h f q$ deletion derivative of this strain grew on minimal succinate medium (failure of RyhB to repress $s d h$ ) and formed blue colonies on minimal medium containing glucose, ampicillin, the $\beta$-galactosidase indicator X-Gal, and the inducer IPTG (failure of OmrB to repress cirA-lacZ).

We grew strain MG1193 pBR-plac-OmrB in the presence or absence of the base analog 2-aminopurine, selected for mutants able to grow on minimal medium containing succinate as the sole carbon source, and then screened those mutants for the ability to form blue colonies on minimal glucose plates containing ampicillin, IPTG, and X-Gal. In the absence of mutagen, only one of the six candidates obtained from the selection on minimal succinate plates was able to form blue colonies on the X-Gal-containing plates; in the presence of mutagen, thousands of colonies were obtained from the selection, and 26 of the 29 candidates screened for expression of the cirA' -'lac $Z$ fusion formed blue colonies. The spontaneous mutant and the 26 mutants generated from chemical mutagenesis that showed a defect in the post-transcriptional regulation of both $s d h C D A B$ by RyhB and cirA by OmrB were further characterized by genetic mapping.

\section{Mapping the mutations responsible for defects in SRNA regulation}

As noted above, $h f q$ mutants were expected from our selection and screen. The set of 27 mutants was screened for $h f q$ alleles by testing for linkage of the mutant phenotype to a yjfP::cat mutation, located $\sim 16 \mathrm{~kb}$ from $h f q$, by $\mathrm{P} 1$ transduction. P1 grown on an $h f q^{+}$yjfP::cat donor was used to transduce the mutants, selecting for chloramphenicol resistance on minimal glucose plates and screening several transductants for loss of the mutant phenotype, that is, for restoration of the negative regulation of $s d h C D A B$ by RyhB (inability to grow on minimal plates containing succinate as the sole carbon source).

The spontaneous mutant and eight of 26 of the mutants that resulted from the chemical mutagenesis had a mutation in the vicinity of $h f q$ that was necessary for the observed defect in sRNA regulation. Sequencing confirmed that all of these strains had mutations in $h f q$ (Table 1). All but one of these mutations resulted in an amino acid substitution in one of the conserved residues on the proximal face of $\mathrm{Hfq}$ that are important for RNA binding (Mikulecky et al. 2004; Sun and Wartell 2006). The remaining mutation in $h f q$ was silent and likely affects the efficiency of translation of this protein. Western blots were performed on crude extract from these mutants and the wild-type strain, and less Hfq was detected in the extracts prepared from all but two mutants, reflecting either unstable $\mathrm{Hfq}$ or less efficient detection of the mutant Hfq with the polyclonal antibody. Higher levels of Hfq were detected in the crude extracts from the mutant expressing an Hfq with an R16C substitution (Fig. 1). Since our primary focus was to identify 
TABLE 1. Mutants obtained from the combined genetic selection and screen

\begin{tabular}{lllccl}
\hline $\begin{array}{l}\text { Gene } \\
\text { mutated }\end{array}$ & Mutation & $\begin{array}{c}\text { Amino acid } \\
\text { change }\end{array}$ & Pool & $\begin{array}{c}\text { Number } \\
\text { obtained } \\
\text { in pool }\end{array}$ & \multicolumn{1}{c}{ Strain(s) } \\
\hline$h f q$ & G12A & None & 1 & 1 & NRD304 \\
$h f q$ & A23G & Gln8Arg & 1 & 1 & NRD307 \\
$h f q$ & A26C & Asp9Ala & $U^{\text {a }}$ & 1 & NRD323 \\
$h f q$ & C46T & Arg16Cys & 3 & 1 & NRD317 \\
$h f q$ & G118A & Asp40Asn & 2 & 1 & NRD310 \\
$h f q$ & T124C & Phe42Leu & 2 & 1 & NRD309 \\
$h f q$ & A164G & Tyr55Cys & 3 & 1 & NRD314 \\
$h f q$ & A170G & His57Arg & 2 AND 3 & 1 & NRD315/NRD325 \\
$p n p$ & C277T & Arg93Cys & 1 AND 2 & 5 & NRD301/NRD302/NRD303/ \\
& & & & & NRD306/NRD312 \\
$p n p$ & C943T & Arg315Cys & 3 & 1 & NRD319 \\
$p n p$ & G1307A & Gly436Asp & 2 & 1 & NRD313 \\
$p n p$ & C1310T & Ser437Phe & 3 & 1 & NRD318 \\
$p n p$ & G1466A & Gly489Asp & 3 & 5 & NRD316/NRD320/NRD321/ \\
& & & & & NRD322/NRD326 \\
$p c n B$ & G196A & Gly66Ser & 1 & 1 & NRD305 \\
$p c n B$ & G209A & Arg70His & 1 & 1 & NRD324 \\
$p c n B$ & C313T & Arg105Cys & 2 & 1 & NRD311 \\
$p c n B$ & C733T & Arg245Cys & 2 & 1 & NRD308 \\
\hline
\end{tabular}

a Unmutagenized pool.

that two independent mutations were present in these strains. The simplest explanation is that the $p c n B$ mutations reduced expression of OmrB by lowering the copy number of the plasmid from which it was being expressed, although this has not been confirmed. Since these $p c n B$ mutants were not generally defective for the post-transcriptional regulation of gene expression by sRNAs, we did not characterize them further.

Thirteen of the mutants obtained from chemical mutagenesis had a mutation necessary for both the defect in the regulation of cirA by $\mathrm{OmrB}$ and the defect in regulation of $s d h C D A B$ by RyhB in the same region of the E. coli chromosome, far from $h f q$ and genetically linked to transposon insertions in the genes $y h b X, y r a H, y r a N$, or $y r a P$. A number of genes implicated in RNA metabolism were in this region of the chromosome, but sequencing of the mutants revealed that the 13 mutants all

genes other than $h f q$ that are required for the posttranscriptional regulation of gene expression by sRNAs, we did not further characterize these mutants.

To map the mutation(s) responsible for the defect in sRNA regulation in the remaining 18 mutants, we first identified a transposon insertion linked to the mutants, sequenced the transposon insertion site, and used this information to search candidate genes nearby for mutations. The details are described in Materials and Methods. Using this mapping strategy, three of the mutants that were obtained from chemical mutagenesis, NRD305, NRD311, and NRD324, had a mutation necessary for the $\mathrm{Lac}^{+}$phenotype that was genetically linked to a transposon insertion between $f h u B$ and hemL, near $p c n B$, which encodes enzyme poly(A) polymerase. Sequencing revealed that NRD305, NRD311, NRD324, and an additional mutant, NRD308, had missense mutations in $p c n B$ (Table 1). Poly(A) polymerase catalyzes the polyadenylation of the $3^{\prime}$ end of mRNAs, which decreases the stability of mRNAs. Mutations in $p c n B$ have previously been implicated in reducing the copy number of plasmids (Lopilato et al. 1986; Liu and Parkinson 1989; March et al. 1989).

However, for these strains, transduction to replace the mutant $p c n B$ allele with a wild-type gene restored the $\mathrm{Lac}^{-}$ phenotype but did not restore the negative regulation of $s d h C D A B$ by RyhB. There was also no difference between a wild-type strain and a $p c n B$ mutant in $\operatorname{sod} B$ turnover upon RyhB induction (data not shown). Thus, the linked mutation was necessary for the defect in the negative regulation of cirA by OmrB but not necessary for the defect in the regulation of the $s d h C D A B$ mRNA by RyhB, suggesting contained one of five different mutations in $p n p$, encoding polynucleotide phosphorylase (PNPase) (Table 1). PNPase is a $3^{\prime}$ to $5^{\prime}$ exoribonuclease that degrades single-stranded RNA and binds directly to Hfq (Mohanty et al. 2004), the RNA helicase RhlB (Liou et al. 2002), and the endonuclease RNase E (Carpousis et al. 1994; Py et al. 1994). PNPase binds to a C-terminal unstructured region of RNase $\mathrm{E}$ adjacent to the binding site for RhlB (Vanzo et al. 1998). Together, these three proteins, PNPase, RhlB, and RNase E, along with a fourth protein, enolase, form the RNA degradosome (Miczak et al. 1996; Py et al. 1996). In E. coli, polynucleotide phosphorylase is essential at low temperatures (Luttinger et al. 1996) and is up-regulated upon cold shock. All of the substitutions occurred in the N-terminal domain of PNPase in residues that are highly conserved among plants and bacteria (Jarrige et al. 2002).

The genetic analysis described above demonstrated that the pnp alleles were necessary for both growth on succinate and the $\mathrm{Lac}^{+}$phenotype, since bringing in the wild-type allele reversed both phenotypes. To determine whether the pnp mutations were sufficient to cause a defect in the posttranscriptional regulation of gene expression by sRNAs, three of the mutant alleles, R93C, R315C, and G436D, as well as a deletion of $p n p$, were introduced into the unmutagenized parent strain, MG1193, harboring pBRplac-OmrB. Introduction of each of these mutations was sufficient to produce the defect in the post-transcriptional regulation of the cirA-lac $Z$ fusion by OmrB, as judged by the formation of red colonies on MacConkey-lactose plates containing ampicillin and chloramphenicol but was not sufficient to allow growth on minimal plates containing 
A.

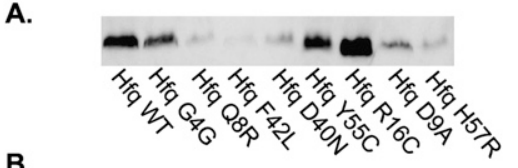

B.

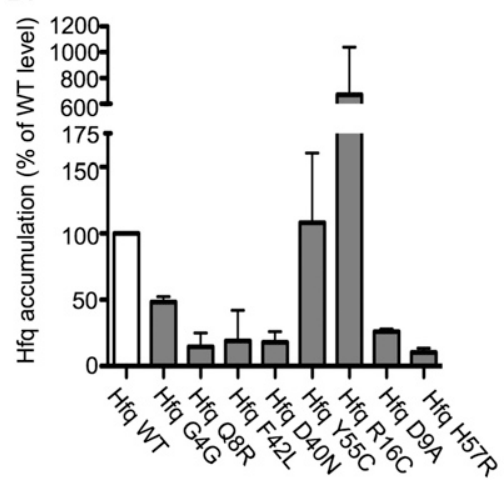

FIGURE 1. Western blot analysis of Hfq in the mutants obtained from the combined genetic selection/screen. The wild-type strain MG1193 (Hfq WT) and the $h f q$ point mutants obtained from the combined genetic selection and screen, NRD304 (Hfq G4G), NRD307 (Hfq Q8R), NRD309 (Hfq F42L), NRD310 (D40N), NRD314 (Y55C), NRD317 (R16C), NRD323 (D9A), and NRD325 (H57R), were grown overnight in glucose minimal M63 medium. A sample was removed from each culture and processed for Western blotting with anti-Hfa polyclonal antibody as described in Materials and Methods. An equal amount of protein based on the cell density of each culture was applied to the polyacrylamide gel. A representative Western blot is shown in $A$. The intensity of the bands on the Western blots was quantified using the Multi Gauge software; intensity of Hfq from the wild-type strain was set to $100 \%$, and the signal from all other strains was normalized to this sample. The results shown in $B$ represent the mean of two experiments.

succinate as the sole carbon source. The best explanation for why pnp mutations were found in the selection would be that the original mutants, selected for growth on succinate, harbored a second mutation that improved growth on succinate minimal plates, possibly by increasing expression of $s d h C D A B$. The second mutation would have arisen in pnp mutant cells, for instance, if these were able to form microcolonies on succinate minimal plates. Experiments described below confirmed that the pnp mutations do compromise RyhB-dependent regulation.

Because a deletion of $p n p$ can abolish OmrB regulation

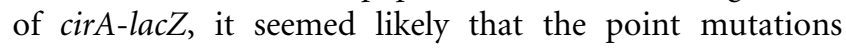
arising from the screen were loss-of-function, rather than gain-of-function, mutations. This was confirmed for a known phenotype of pnp mutants, cold sensitivity (Luttinger et al. 1996; Zhou and Deutscher 1997). Each of the pnp mutations isolated, as well as a $p n p$ deletion, were transduced into strain DJ624 and tested for growth at $37^{\circ} \mathrm{C}$ and $25^{\circ} \mathrm{C}$ on LB plates. The pnp mutants grew significantly slower than the wild type at $37^{\circ} \mathrm{C}$ and showed no growth after $2 \mathrm{~d}$ of incubation at $25^{\circ} \mathrm{C}$, whereas the wild-type strain grew well at $25^{\circ} \mathrm{C}$ (Fig. 2A). The cold sensitivity of the pnp point mutants or pnp deletion mutants was complemented by expression of wild-type $p n p$ from a plasmid (Fig. 2B). These results demonstrated that the pnp point mutants had phenotypes similar to $p n p$ null mutants and were recessive to the wild-type $p n p$.

\section{pnp mutations have a general effect on sRNA stability}

The isolation of multiple loss-of-function mutations in $p n p$ that disrupted sRNA function was unexpected, and the step at which PNPase might act was not immediately apparent. For sRNAs to effectively regulate mRNA targets, they need to accumulate to a level sufficient for activity, interact with $\mathrm{Hfq}$, and, in a process that is not fully understood, interact with their target mRNAs in an Hfq-dependent manner. The interaction between the sRNA and mRNA via base-pairing can lead to changes in translation and/or to changes in mRNA stability.

The first step that was analyzed was sRNA accumulation in $p n p$ mutants. In wild-type cells, sRNAs accumulate rapidly after induction and are stable when new transcription is blocked, for instance, with rifampicin. However, in the absence of $\mathrm{Hfq}$, they are unstable even when transcription

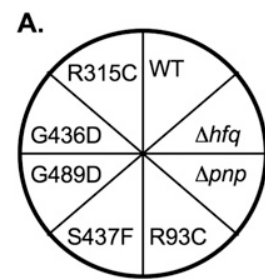

B.

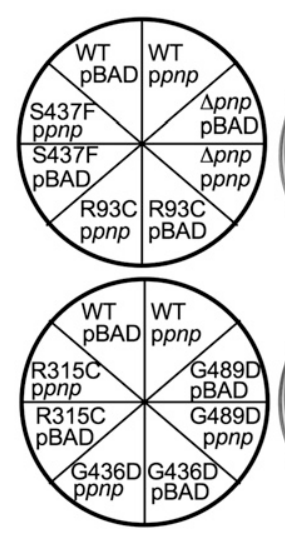

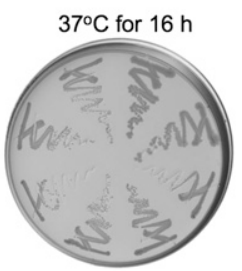

LB at $25^{\circ} \mathrm{C}$

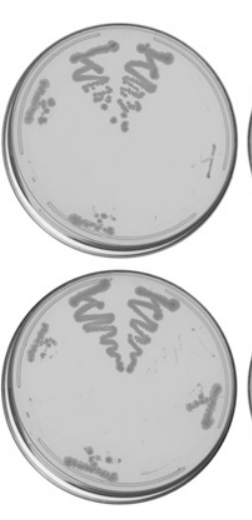

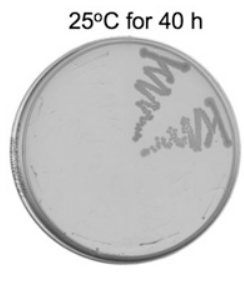

LB + arabinose at $25^{\circ} \mathrm{C}$

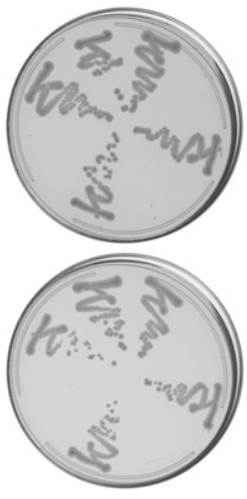

FIGURE 2. The growth of the $p n p$ mutants isolated in the combined genetic selection and screen at $37^{\circ} \mathrm{C}$ and $25^{\circ} \mathrm{C}$. (A) Strains DJ624 (WT), or isogenic derivatives harboring a $\Delta h f q$ mutation (NRD459), a $\Delta p n p$ mutation (NRD473), or a point mutation in $p n p$ that encodes a PNPase with an R93C (NRD453), S437F (NRD457), G489D (NRD493), G436D (NRD494), or an R315C (NRD495) substitution were streaked onto two LB plates. One plate was incubated at $37^{\circ} \mathrm{C}$ for $16 \mathrm{~h}$, and the other was incubated at $25^{\circ} \mathrm{C}$ for $40 \mathrm{~h}$. (B) The wild-type strain or the pnp mutants from $A$ were transformed with pBAD30 (pBAD) or a plasmid derivative that expresses PNPase from an arabinose-inducible promoter (pNRD412; ppnp), streaked out onto an LB plate containing ampicillin or an LB plate containing ampicillin and arabinose at a final concentration of $0.1 \%$, and then incubated at $25^{\circ} \mathrm{C}$ for $48 \mathrm{~h}$. The results are representative of three experiments. 
is blocked, suggesting that Hfa protects them from ribonucleases (RNase E in the tested cases [Massé et al. 2003]). However, if their half-life is monitored while active cellular transcription proceeds (for instance, when new sRNA synthesis is turned off at the level of the sRNA promoter), they are unstable even in $\mathrm{hfq}^{+}$cells (Massé et al. 2003). Therefore, Hfq protects sRNAs from degradation, and that protection is apparently abrogated upon pairing. If sRNAs are not properly bound to Hfq, or if Hfq were not able to protect them from degradation, we would expect more rapid degradation of the sRNAs and, therefore, less accumulation, possibly reducing the sRNA levels below that needed for effective regulation.

We examined the accumulation and stability of three Hfq-binding sRNAs: RyhB, SgrS, and CyaR. While RyhB accumulation and activity were monitored in the original genetic selection, the other two sRNAs were not. However, we found that each of these sRNAs was more unstable in the $p n p$ mutants than in a wild-type strain (Fig. 3).

RyhB accumulation and stability were determined by growing cells in LB to mid-log phase and adding dipyridyl to chelate $\mathrm{Fe}$ and inactivate the Fur repressor. Samples were taken to monitor RyhB synthesis. Rifampicin was added 16 min after dipyridyl treatment to stop all new synthesis, and samples were taken to monitor decay of the sRNA. As expected, RyhB was rapidly induced to a high level in the wild-type strain and to a much lower level in the $h f q$ mutant ( $40 \%$ of wild type). The level of RyhB was even lower in the pnp mutants (21\%-24\% of wild-type for two mutants) (Fig. $4 \mathrm{~A})$. Turnover of RyhB after rifampicin treatment was rapid in the $h f q$ mutant and slow in the wild-type strain, as previously seen (Massé et al. 2003). In both the pnp deletion and the pnp G463D mutants, RyhB turnover was significantly faster than in the wild-type strain (Fig. 3A).

The next sRNA tested, SgrS, is induced upon accumulation of glucose-phosphate or after addition of methyl$\alpha$-D-glucopyranoside $(\alpha-M G)$ to cells. SgrS induction leads to down-regulation of $p t s G$, encoding the glucose transporter (Vanderpool and Gottesman 2004). The experiment was similar to that described above, except that methyl$\alpha$-D-glucopyranoside $(\alpha-M G)$ was added to induce SgrS expression. Quantification of the Northern blots shown in Figure 4B revealed that SgrS levels after 15 min induction with $\alpha$-MG were reproducibly $35 \%$ lower in the $h f q$ mutant than the wild-type strain. This decrease in SgrS levels in the $h f q$ mutant was less than that observed by Kawamoto et al. (2005); there are differences between their work and ours in the genetic background used, as well as in the growth phase, induction time, and amount of inducer $(\alpha-M G)$. Regardless, the half-life of SgrS was significantly reduced in the pnp point mutant and the pnp deletion mutant as compared to the wild-type strain but was lowest in the $h f q$ mutant (Fig. 3B).

The effect of pnp mutants on the stability of a third sRNA, CyaR, was also determined. While CyaR is regulated
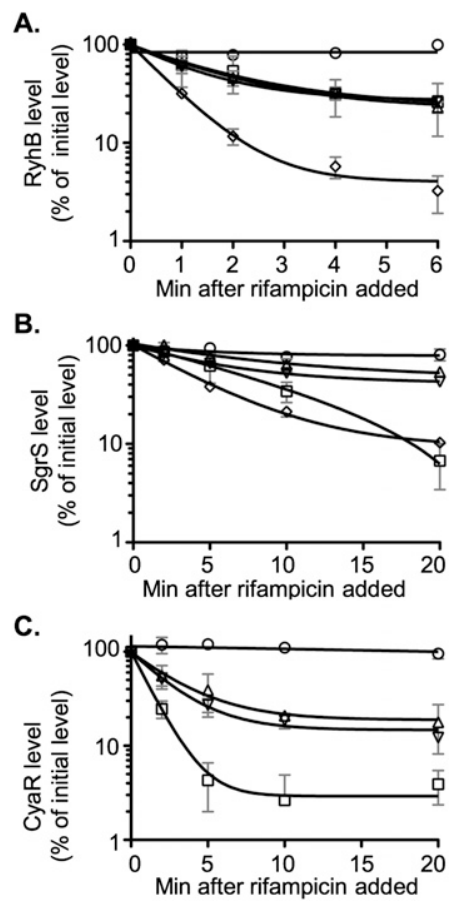

FIGURE 3. Northern blot analysis of RyhB, SgrS, and CyaR turnover in pnp mutants. $(A, B)$ Overnight cultures of the wild-type strain DJ624 (circles), the $h f q$ deletion mutant NRD459 (squares), the pnp deletion mutant NRD473 (upward pointing triangles), the pnp point mutant NRD494 (downward pointing triangles), or the $\Delta p n p \Delta h f q$ double mutant NRD535 (diamonds) were diluted 200-fold into fresh LB. (C) Overnight cultures of a cyaR deletion strain (NRD531; circles), or isogenic derivatives carrying an $h f q$ deletion (NRD532; squares), a $p n p$ deletion (NRD533; upward pointing triangles), or a $p n p$ point mutation (NRD534; downward pointing triangles), all harboring a plasmid expressing CyaR from a lac promoter (pNRD405), were diluted 200 -fold in fresh LB containing ampicillin. All strains were grown at $37^{\circ} \mathrm{C}$ to an $\mathrm{OD}_{600}$ between 0.3 and 0.4 , and dipyridyl $(A)$, $\alpha-\mathrm{MG}(B)$, or IPTG $(C)$ was then added to each culture to induce sRNA synthesis. A sample was taken from each culture 15 min after induction. Sixteen minutes after induction, rifampicin was added to each culture, and additional samples were taken at the indicated times. RNA was extracted, fractionated on a polyacrylamide gel, and transferred to a nylon membrane. The Northern blot was developed using the $5^{\prime}$-biotinylated RyhB probe $(A), \operatorname{SgrS}$ probe $(B)$, or CyaR probe $(C)$ as described in Materials and Methods. The intensity of the bands in the Northern blots was quantified using the Multi Gauge software. The band intensity for the 0 -min sample was set to $100 \%$, and other samples were normalized to the 0 -min sample. The results represent the mean of two experiments, and the standard deviation is indicated by the light gray bars.

by CRP and cAMP, in this case, CyaR was expressed from an inducible lac promoter on a plasmid. As for the experiments above, induction with IPTG was for $15 \mathrm{~min}$, and then the cells were treated with rifampicin. As observed for RyhB and SgrS, CyaR was least stable in the $h f q$ mutant but was less stable in the pnp mutants than in the wild-type strain (Fig. 3C).

In parallel, the activity of each of these sRNAs was monitored by measuring the level of the mRNAs for one of their targets ( $\operatorname{sodB}$ as a target for RyhB, $p t s G$ as a target for 

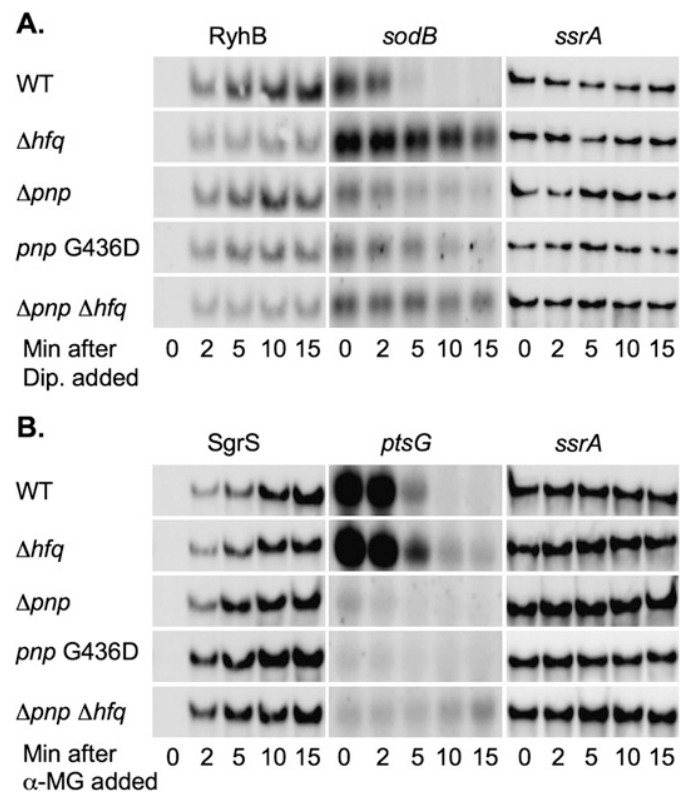

C.

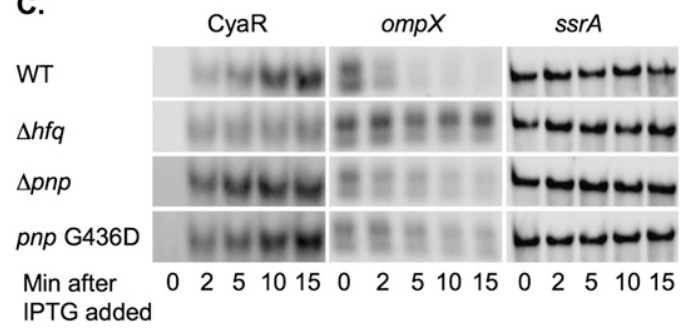

FIGURE 4. Northern blot analysis of $\operatorname{sod} B, p t s G$, and $\operatorname{smp} X$ mRNA fate in pnp mutants. From the same cultures used in Figure 3, samples were removed for RNA isolation from each culture prior to and 2, 5, 10 , and 15 min after dipyridyl $(A), \alpha-\mathrm{MG}(B)$, or IPTG $(C)$ was added to the culture. An equal amount of RNA from each sample was fractionated on a polyacrylamide or agarose gel, transferred to a nylon membrane, and a Northern blot developed using the $5^{\prime}$-biotinylated RyhB and sodB probe $(A)$, SgrS and ptsG probe $(B)$, or CyaR and ompX probe $(C)$ as described in Materials and Methods. The membranes used for the RyhB, SgrS, or CyaR Northern blots were subsequently stripped by boiling in $0.5 \%$ SDS and developed using the ssrA probe as a loading control. The results are representative of two experiments.

SgrS, and ompX as a target for CyaR). For each of these targets, induction of the appropriate sRNA led to rapid degradation of the mRNA in wild-type cells; in $h f q$ mutants, no, or much slower, degradation of the mRNA took place (Fig. 4A,B,C). In the pnp mutants, two effects were apparent. First, unexpectedly, the levels of the mRNAs were significantly decreased before induction of the sRNA (see 0 -min time point, Fig. $4 A, B, C)$. In the case of $\operatorname{sodB}$ and ompX, the mRNAs were approximately one-third lower in the $p n p$ deletion or $p n p$ point mutant as compared to the wild-type strain prior to sRNA induction, whereas $p t s G$ mRNA was less than one-tenth the level of the wild-type strain in the pnp mutants before sRNA induction. Second, in the case of $\operatorname{sodB}$ and $\operatorname{ompX}$, turnover of the mRNAs was reduced in the pnp mutants (Fig. $4 \mathrm{~A}, \mathrm{C}$ ). It was difficult to assess the effect of the pnp mutations on the negative regulation of $p t s G \mathrm{mRNA}$, since all strains showed an initially rapid turnover of the $p t s G$ mRNA after SgrS induction, and the $p t s G$ mRNA level was too low to detect at the time points at which $p t s G$ mRNA stabilized in the $h f q$ mutant (Fig. 4B).

Overall, these experiments pointed to instability of three different sRNAs in the $p n p$ mutants. The decreased stability of the sRNAs may be sufficient to explain the parallel loss in sRNA-dependent regulation. SgrS, which was much more stable in the pnp mutants than RyhB or CyaR and still accumulated to a high level in the $p n p$ mutant, was also still able to carry out some regulation of $p t s G$. This implied an unexpected protective role for PNPase in allowing sRNA accumulation/stabilization.

\section{The defect in SRNA regulation caused by the pnp mutations was independent of $\mathrm{Hfq}$}

As noted above, and as demonstrated by the isolation of $h f q$ mutations, Hfq is known to be necessary for the sRNAs studied here to act. Thus, our initial model was that lossof-function mutations in $p n p$ led to reduced expression of Hfq and, as a consequence, reduced sRNA stability and function. To test this model, we examined the level of Hfq by Western blot in isogenic strains that carry the $p n p$ deletion or one of five $p n p$ missense alleles. Hfq levels were similar in a wild-type strain and in the $p n p$ mutants (Fig. 5A). Therefore, pnp does not act by decreasing Hfq levels.

Another way in which Hfq might be unavailable for binding and stabilizing sRNAs would be if the loss of PNPase increased some Hfq-binding RNA(s), making Hfq limiting and therefore unavailable for other RNAs. If so, overexpression of Hfq should lead to an increase in the stabilization of the sRNAs in the pnp mutant and an increase in the RyhB-stimulated turnover of the $\operatorname{sodB}$ mRNA. Thus, we grew a wild type strain or isogenic derivatives carrying either an $h f q$ or $p n p$ deletion and harboring either a vector or a plasmid containing $h f q$ under the control of an arabinose promoter, induced expression of Hfq with arabinose, and then induced RyhB expression by the addition of dipyridyl to the culture medium. RyhB synthesis and stability and $\operatorname{sodB}$ turnover were assessed as in the experiments described above. Hfq expressed from the plasmid complemented an $h f q$ mutant for $\operatorname{sodB}$ turnover and increased the stability of RyhB, confirming the activity of the plasmid (Fig. 5B,C, cf. lines 3 and 4). Overexpression of $\mathrm{Hfq}$ in wild-type cells increased the stability of RyhB slightly and increased its accumulation significantly (Fig. 5B,C, cf. lines 1 and 2). However, overexpression of Hfq from a plasmid did not suppress the defects in a pnp deletion mutant; RyhB did not accumulate (Fig. 5C, lines 5 and 6) or increase in stability (Fig. 5B), and $\operatorname{sodB}$ did not show any increased 
A.

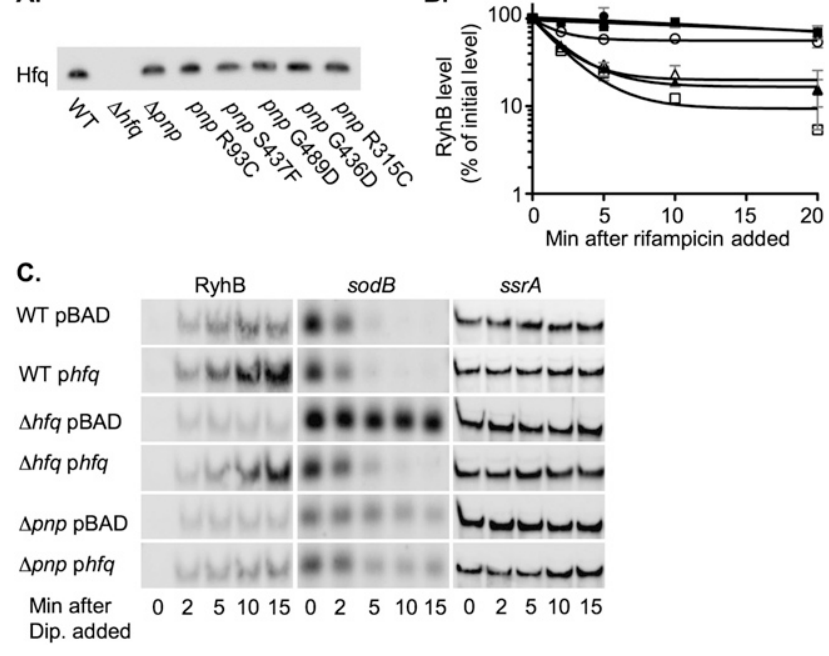

FIGURE 5. Effect of $p n p$ mutations on $\mathrm{Hfq}$ and effects of $\mathrm{Hfq}$ overexpression in $p n p$ mutants. $(A)$ Overnight cultures of the wildtype strain DJ624 or derivatives harboring an $h f q$ deletion (NRD459), pnp deletion (NRD473) or pnp allele encoding a PNPase with an R93C (NRD453), S437F (NRD457), G489D (NRD493), G436D (NRD494), or R315C substitution (NRD495) were diluted 200-fold in fresh LB medium. Cells were grown at $37^{\circ} \mathrm{C}$ to an $\mathrm{OD}_{600}$ of $\sim 1.0$, a sample was taken from each culture, and the protein was TCA-precipitated. An equal amount of protein based on the cell density of each culture at the time of sampling was then processed as in Figure 1. $(B, C)$ An overnight culture of the wild-type strain DJ624 (WT; circles), $h f q$ deletion strain NRD459 ( $\Delta h f q$; squares), or $p n p$ deletion strain NRD473 ( $\Delta p n p$; triangles) harboring pBAD30 (pBAD; open symbols) or a plasmid expressing $h f q$ from an arabinose inducible promoter (pNRD414; phfq; solid symbols) was diluted 200-fold in fresh LB medium containing ampicillin. Each culture was grown to an $\mathrm{OD}_{600}$ of 0.3-0.4, and arabinose was added. After 5 min of induction, a sample was taken from each culture (0-min samples), and then dipyridyl added. Additional samples were taken as indicated. Sixteen minutes after dipyridyl addition, rifampicin was added to the cultures and samples taken 2, 5, 10, and 20 min after rifampicin treatment. The RNA was extracted and processed as described in Figure 4, using the 5 '-biotinylated RyhB, sodB, and ssrA probe. The results in $B$ represent the mean of two experiments, and the standard deviation is indicated by the gray bars. The blots in $A$ and $C$ are representative of two experiments.

degradation (Fig. 5C, cf. lines 5 and 6). Based on these results, we conclude that the defect in the post-transcriptional regulation of gene expression in the pnp null mutants is not due to titration of Hfq.

While Hfq does not appear to be limiting, it seemed possible that lack of PNPase leads to inactivation of Hfq by an unknown mechanism. In this model, the effects of the pnp mutants would be upstream of Hfq, and an $h f q$ mutant would be epistatic to the pnp mutants. However, the halflife of the RyhB and SgrS sRNAs demonstrated additive effects of deleting $p n p$ and $h f q$. The half-lives of RyhB and SgrS were even shorter in the strain harboring both the $h f q$ and $p n p$ deletion than in a strain harboring either deletion alone (Fig. 3A,B). These results suggested that PNPase was not exerting its effect on the regulation of gene expression by sRNAs solely through Hfq.

\section{Suppression of pnp mutant phenotypes by deletion of the C terminus of RNase $E$}

PNPase is known to bind to RNase E, which is essential for the coupled degradation of several sRNAs and their targeted mRNAs (Massé et al. 2003; Morita et al. 2003; Guillier and Gottesman 2008). Therefore, we considered a model in which the decreased stability of the sRNAs is caused by an increased rate of turnover by RNase E. This increased sRNA degradation may in itself be responsible for the defect in sRNA regulation. In this model, PNPase normally decreases the turnover of sRNAs by modulating RNase E activity, or by blocking the binding of Hfq-bound sRNAs to RNase E, thus protecting them from degradation.

rne is essential, so deleting it entirely was not feasible. Given the known interaction of PNPase with the $\mathrm{C}$ terminus of RNase E, we examined the role of the RNase E C terminus in the phenotypes of the pnp mutants. We introduced different alleles of rne, including rne-131, $r n e \Delta 10$, and $r n e \Delta 14$, into a wild-type strain or a $p n p$ deletion mutant. rne-131 encodes an RNase E protein lacking the entire unstructured C-terminal domain involved in binding PNPase, enolase, and RhlB. rne $\Delta 14$ encodes an RNase E that lacks the region that includes the RhlB and enolase binding sites but retains the PNPase binding site (Leroy et al. 2002). rne $\Delta 10$ encodes an RNase E that lacks the region that includes the PNPase binding site but retains the enolase and RhlB site (Leroy et al. 2002). The ability of the resulting strains, an isogenic $r n e^{+} p n p$ deletion mutant and a wild-type strain, to grow at $25^{\circ} \mathrm{C}, 32^{\circ} \mathrm{C}$, and $37^{\circ} \mathrm{C}$ was determined. Unexpectedly, introduction of the $r n e-131$ or $r n e \Delta 14$ allele into the $p n p$ deletion strain partially suppressed the cold sensitivity of the pnp deletion mutant at $32^{\circ} \mathrm{C}$ and $25^{\circ} \mathrm{C}$ and decreased the doubling time of the pnp deletion mutant at $37^{\circ} \mathrm{C}$ (Fig. 6). On the other hand, introduction of the rne $\Delta 10$ allele into the pnp deletion mutant did not suppress the cold sensitivity of the $p n p$ deletion mutant and actually increased the doubling time of the $p n p$ deletion mutant at $37^{\circ} \mathrm{C}$ (Fig. 6). Introduction of any of these rne alleles into a wild-type strain had little or no effect on growth at $25^{\circ} \mathrm{C}, 32^{\circ} \mathrm{C}$, or $37^{\circ} \mathrm{C}$ (Fig. $6 \mathrm{~B}$; data not shown).

Suppression of one phenotype of a pnp deletion, cold sensitivity, by rne-131 or rne $\Delta 14$ suggested that these rne alleles might also suppress another phenotype, the defect in sRNA-dependent regulation. Testing this is complicated by the involvement of RNase $\mathrm{E}$ in degradation of target mRNAs in response to sRNA pairing; because of this, monitoring mRNA levels to evaluate sRNA function would not have been informative (Massé et al. 2003; Morita et al. 2005). To avoid this complication, we took advantage of the observation that translation of mRNAs can be inhibited by sRNAs, even in the absence of mRNA degradation (Maki et al. 2008).

As described above, $\operatorname{omp} X$ is negatively regulated by CyaR. While this regulation can be seen at the level of 


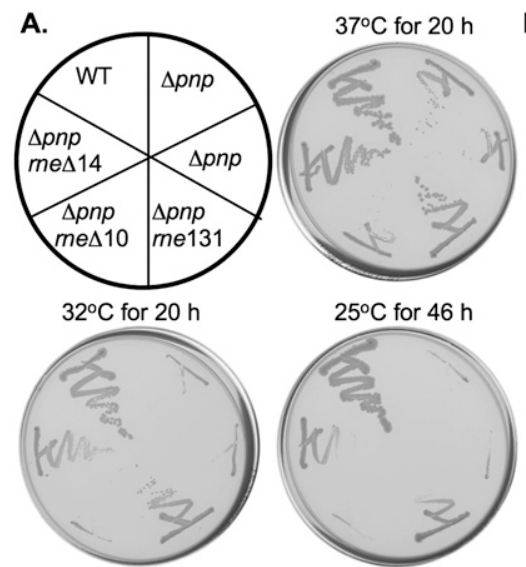

B.

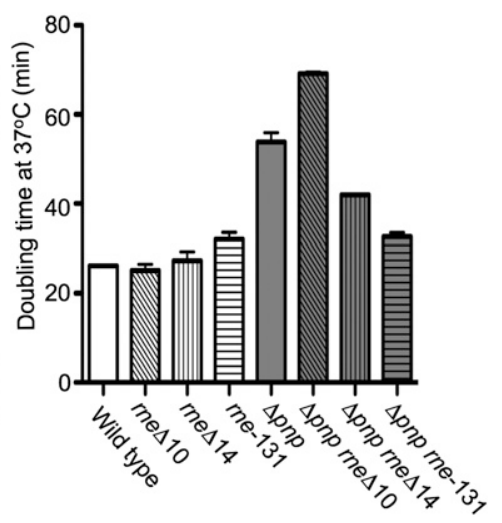

FIGURE 6. The effect of rne truncations on growth of a pnp deletion mutant. (A) A wild-type strain (DJ624; WT) or isogenic derivatives harboring a pnp deletion (NRD473 and NRD579; $\Delta p n p)$ or a $p n p$ deletion and an rne allele truncating the C-terminal domain of rne were streaked onto $\mathrm{LB}$ plates that were incubated at $37^{\circ} \mathrm{C}, 32^{\circ} \mathrm{C}$, or $25^{\circ} \mathrm{C}$. The rne- 131 allele encodes an RNase $\mathrm{E}$ that lacks residues 585-1061, the complete unstructured C-terminal domain (NRD578; $\Delta p n p$ rne-131); rne $\Delta 10$ lacks residues 844 to 1045 , a region that includes the PNPase binding site (NRD 585; $\Delta p n p$ rne $\Delta 10$ ); rne $\Delta 14$ lacks residues 636 to 845 , a region that includes the binding site for RhlB and enolase (NRD589; $\Delta p n p$ rne $\Delta 14$ ). (B) Overnight cultures of the wild-type (wild type) and $p n p$ deletion strain $(\Delta p n p)$ harboring the wild-type rne allele or the truncated rne alleles listed in $A$ were diluted 200 -fold into fresh LB liquid medium and incubated at $37^{\circ} \mathrm{C}$ and the doubling time during exponential growth determined. These results represent the mean from two independent experiments.

mRNA instability (Johansen et al. 2008; Papenfort et al. 2008; De Lay and Gottesman 2009), mutations such as rne131 block mRNA degradation (Fig. 7A,B). Therefore, we instead evaluated an ompX-lac $Z$ translational fusion, also negatively regulated by CyaR (Figs. 7B, 8A). In cells expressing CyaR from a plasmid, expression of the fusion was reduced by $>60 \%$, and this regulation was not perturbed in an rne-131 mutant cell (Fig. 8A). In parallel with the failure of full regulation of ompX mRNA or the omp $X^{\prime}-$ - lac $Z^{\prime}$ mRNA in the $p n p$ deletion strain, expression of the fusion was reduced by $<40 \%$ upon CyaR expression in the $\Delta p n p$ strain (Fig. 8A). Thus, deletion of pnp partially disrupts the negative regulation of $m_{p} X$ mRNA by CyaR. Introduction of the rne-131 allele into the pnp mutant completely suppressed the defect in sRNA regulation, while introduction of the rne $\Delta 14$ allele resulted in partial suppression (Fig. $8 \mathrm{~A}$ ). In contrast, introduction of the rne $\Delta 10$ allelle into the $p n p$ mutant did not suppress the defect in the regulation of ompX by CyaR but instead exacerbated the loss of regulation (Fig. 8B).

Consistent with the pnp mutant acting to perturb RNase E-dependent turnover of mRNAs and sRNAs, the rne-131 mutation was epistatic to a deletion of pnp in terms of CyaR accumulation and stability and degradation of either ompX or ompX-lac Z mRNAs. As described above, CyaR was relatively stable in wild-type strains; it was slightly more stable in the rne-131 mutant host (Fig. 7C, cf. X's and circles). As noted above, CyaR was very unstable in a $p n p$ mutant. Introduction of the rne-131 allele into the pnp deletion increased the stability of the CyaR sRNA to a level similar to that seen in the rne-131 mutant alone (Fig. 7C, stars). This increased stability was also reflected in the increased accumulation of CyaR in this double mutant (data not shown).

The rapid turnover of the $\operatorname{omp} X$ and omp $X^{\prime}$-'lac $Z$ mRNA after CyaR induction seen in a wild-type strain was significantly decreased in the rne-131 host (Fig. 7A,B). In this case, both the pnp mutation and the rne-131 mutation led to slower turnover of ompX RNA, so it is more difficult to judge epistasis. However, the double mutant $(\Delta p n p$ rne-131) behaved very similarly to the rne-131 mutation, in terms of the turnover of both ompX and ompX'-'lacZ RNA (Fig. 7A,B). Coupled with the suppression of translational inhibition (Fig. 8A), these results suggested that restoring CyaR stability and accumulation was sufficient to restore the function of the sRNA to a pnp mutant. Turnover of the mRNA was a second level of regulation and was abrogated or decreased by the rne-131 allele.

\section{Positive regulation was also defective in a pnp mutant and suppressed by mutations in rne}

sRNAs can positively regulate gene expression as well as negatively regulate it. If $p n p$ mutants disrupt sRNA function by reducing sRNA stability, we would expect a role for PNPase in positive regulation as well, and we would predict that the C-terminal deletions in rne should suppress the $p n p$ mutant effect. To determine if PNPase also is required for positive regulation, we examined the effect of the $p n p$ deletion on RpoS expression; RpoS translation is positively regulated by DsrA (Lease et al. 1998; Majdalani et al. 1998) and RprA (Majdalani et al. 2001) and is dependent upon Hfq.

A set of isogenic strains (wild type, pnp, rne $\Delta 14$, rne-131, pnp rne $\Delta 14$, and pnp rne-131) harboring a vector or a plasmid that expressed either DsrA or RprA from a lac promoter were grown to an $\mathrm{OD}_{600}$ of $\sim 0.3$, DsrA or RprA expression was induced for $20 \mathrm{~min}$, and then samples were taken from each culture. The relative levels of RpoS in each strain, with either a vector or the sRNA-expressing plasmids, were analyzed by Western blot (Fig. 9). When DsrA or RprA were overexpressed in the pnp deletion mutant, RpoS accumulated to a level that was less than one-fifth of the RpoS level observed in the wild-type strain (Fig. 9A,B), indicating that loss of $p n p$ results in the decreased expression of at least one gene positively regulated by sRNAs. 
A.

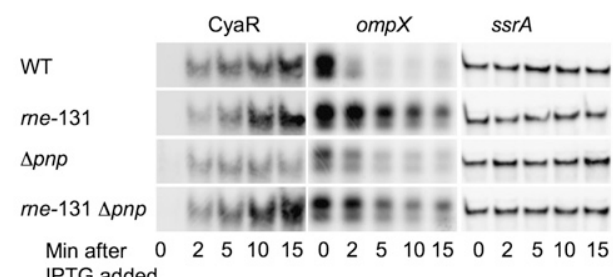
IPTG added

B.

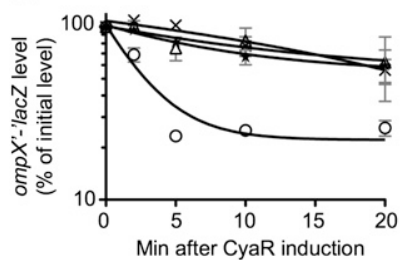

C.

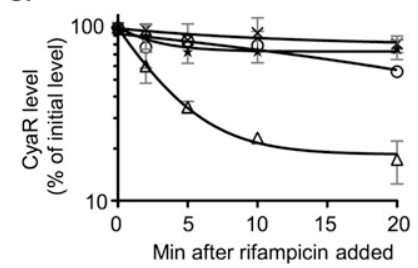

FIGURE 7. Analysis of the effect of a pnp deletion and the rne-131 allele on CyaR and ompX turnover. $(A, B, C)$ Overnight cultures of the cyaR deletion strain (NRD377; circles or WT), an rne-131 mutant (NRD674; X's or rne-131), a pnp deletion mutant (NRD677; triangles or $\Delta p n p$ ), and an rne-131 $\Delta p n p$ double mutant (NRD680; stars or $\Delta p n p$ rne-131) harboring a plasmid expressing CyaR from a lac promoter (pNRD405) were diluted 200-fold in fresh LB containing ampicillin. Cultures were grown at $37^{\circ} \mathrm{C}$ to an $\mathrm{OD}_{600}$ between 0.3 and 0.4 , a sample was removed, and IPTG was then added to each culture. Additional samples were taken 2, 5, 10, and $15 \mathrm{~min}$ after IPTG addition. Sixteen minutes after IPTG was added to each culture, rifampicin was added to each culture, and samples were taken $2,5,10$, and $20 \mathrm{~min}$ after rifampicin treatment. The RNA was extracted and processed as described in Figure 4 using the CyaR, ompX, and ssrA probe $(A)$, lacZ probe $(B)$, or CyaR probe $(C)$. The results in $A$ are representative of two experiments. The intensity of the bands in the Northern blots in $B$ and $C$ was quantified using the Multi Gauge software. The band intensity for the 0 -min sample was set to $100 \%$, and each additional sample was normalized to the 0 -min sample. The results in $B$ and $C$ represent the mean of two experiments, and the standard deviation is indicated by the gray bars.

Mutations rne $\Delta 14$ or rne-131 alone increased expression of RpoS upon DsrA induction (2.5-fold or 2.2-fold) and upon RprA induction (5.6-fold or 3.9-fold), presumably reflecting less rapid turnover of the rpoS mRNA and possibly higher levels of accumulation of the sRNAs (Fig. 9; McCullen et al. 2010). Nonetheless, it was apparent that the rne alleles suppressed the pnp deletion. As for regulation of the ompX::lacZ fusion, the rne mutants were epistatic to the pnp mutants for expression of RpoS. The very low RpoS expression in the pnp mutant was increased in an rne-131 pnp strain to a level 1.5-fold (for DsrA) or 2.1-fold (for RprA) higher, respectively, than the wild-type strain overexpressing DsrA or RprA and only modestly lower than was seen in the $p n p^{+}$rne strains (Fig. 9). Similarly, introduction of the rne $\Delta 14$ mutation into the pnp mutant increased the expression of RpoS to a level higher than the wild-type strain, i.e., 1.5-fold higher upon DsrA induction and 2.1fold higher upon RprA induction (Fig. 9). Basal levels of RpoS in the presence of vector were much lower but show a similar pattern to that seen after sRNA expression (low in the pnp mutant, higher in the pnp rne double mutants), presumably reflecting the effects of these mutations on endogenous sRNAs (Fig. 9, vector panels).

Altogether, these results demonstrate that the complete truncation of the $\mathrm{C}$ terminus of RNase $\mathrm{E}$ or deletion of a region of RNase $\mathrm{E}$ that includes the RhlB and enolase binding sites suppresses the cold sensitivity and the defect in sRNA regulation observed in the pnp mutant.

\section{Increased expression of RNase E was not sufficient to cause a defect in SRNA regulation}

One possible explanation for our observations was that there was a higher level of RNase $\mathrm{E}$ in the pnp mutants and that the higher concentration of RNase E in the cell led to more rapid turnover of sRNAs. In this model, introduction of the rne-131 and rne $\Delta 14$ alleles into the pnp mutant counteracts this effect, because the RNase E encoded by these alleles has reduced activity, at least for some substrates (Leroy et al. 2002). In fact, RNase E protein levels were moderately increased in a pnp mutant (Fig. 10A,B). However, if this was the full explanation of the $\Delta p n p$ phenotype, we would expect overexpression of RNase E to mimic the effect of a pnp null mutation. This was not the case. When RNase E was overexpressed to a level far higher $(15-30 \times$ greater than wild type) than observed in the pnp deletion mutant (Fig. 10C), the half-life of RyhB decreased but was still significantly higher than what was observed in the pnp mutant (Fig. 10D, cf. Fig. 3A). More importantly, the RyhB-mediated regulation of $\operatorname{sodB}$ mRNA was not affected by the overproduction of the RNase E (Fig. 10E). Therefore, we conclude that the pnp mutant has effects beyond the somewhat increased level of RNase E. An alternative model is considered below.

\section{DISCUSSION}

We have developed and validated a novel genetic approach for identifying genes required for the post-transcriptional regulation of gene expression by sRNAs in E. coli. This combined genetic selection and screen facilitates the isolation of mutants defective in sRNA regulation. The validity of this approach was demonstrated by the isolation of several mutants defective for sRNA regulation that had mutations in $h f q$, an RNA chaperone that is essential for the posttranscriptional regulation of gene expression by sRNAs. By using chemical mutagenesis rather than transposon insertion mutagenesis, we hoped to isolate point mutations in genes encoding both essential and non-essential proteins involved in sRNA function. While many of the mutants that we obtained had mutations in $h f q$, most had mutations in $p n p$, encoding polynucleotide phosphorylase.

\section{Nature of $h f q$ mutations}

In all but one case, the $h f q$ mutations led to amino acid substitutions in highly conserved residues on the proximal 
A.
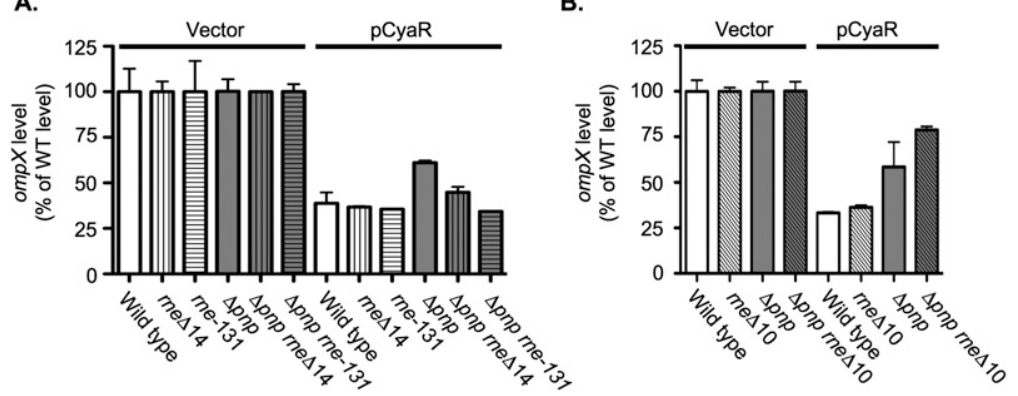

FIGURE 8. Analysis of the effect of a $p n p$ deletion and the rnes14 and rne-131 alleles on sRNA regulation. (A) Overnight cultures of the cyaR deletion strain (NRD377; wild type), an rne $\Delta 14$ mutant (NRD673; rne $\Delta 14$ ), an rne-131 mutant (NRD674; rne-131), a pnp deletion

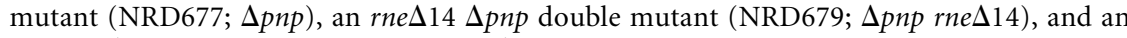
$r n e-131 \Delta p n p$ double mutant (NRD680; $\Delta p n p$ rne-131) harboring either a vector or a plasmid expressing CyaR from a lac promoter (pNRD405) were diluted 200-fold in fresh LB containing ampicillin and IPTG. (B) Overnight cultures of the cyaR deletion strain (NRD377; wild type), an rne $\Delta 10$ mutant (NRD672; rne $\Delta 10)$, a pnp deletion mutant (NRD677; $\Delta p n p)$, and an rne $\Delta 10 \Delta p n p$ double mutant (NRD678; $\Delta p n p$ rne $\Delta 10)$ harboring either a vector or a plasmid expressing CyaR from a lac promoter (pNRD405) were diluted 200-fold in fresh LB containing ampicillin and IPTG. Strains were grown to an $\mathrm{OD}_{600}$ between 0.3 and 0.4. Samples were taken from all cultures, and a $\beta$-galactosidase assay was performed on those samples as described by Miller (1992).

face of Hfq, including Q8, D9, R16, D40, F42, Y55, and H57. Measurements of Hfq levels suggest lower levels of protein in most of the mutants and unexpectedly higher levels in R16C (Fig. 1). In vitro, alanine substitutions at many of these residues have been previously shown to reduce the affinity of $\mathrm{Hfq}$ for poly-A RNAs or DsrA (Mikulecky et al. 2004; Sun and Wartell 2006). A D40A substitution has been shown to destabilize the protein (Mikulecky et al. 2004). Thus, these $h f q$ mutants may be defective in sRNA regulation both because the Hfq protein was destabilized and because the Hfq that was expressed had a low affinity for sRNA binding. Another $h f q$ mutant that we obtained expressed an Hfq with a D9A substitution. Hfq D9A was previously shown to bind a $A_{5} G$ RNA, DsrA, and rpoS mRNA in vitro with higher affinity than the wild-type Hfq (Mikulecky et al. 2004). The basis for the defect in sRNA regulation in this mutant is not known, and its isolation as defective in sRNA function suggests that it may be interesting to investigate further. Perhaps too tight binding to the sRNA or mRNA is detrimental to pairing. One mutant, NRD304, showed the expected linkage for an $h f q$ mutation, but the only change seen was silent, changing the third codon in $h f q$ from a GGG codon to GGA. We note that the DNA sequence of the first 6 codons of $h f q$ are highly conserved (data not shown). This substitution of the third codon GGG to GGA in $h f q$ reduced the expression of Hfq, although whether that reduction is sufficient to explain the phenotype is not known (Fig. 1). This is the first direct selection of point mutants in $h f q$ that we are aware of, but the overlap with previously studied sitedirected mutations suggests that most critical sites have previously been investigated by site-directed mutagenesis.

\section{PNPase mutants}

Half of the mutants isolated and characterized had mutations in pnp. PNPase is a $3^{\prime}-5^{\prime}$ exoribonuclease, which binds directly to the C-terminal scaffold domain of RNase E (Carpousis et al. 1994; Py et al. 1994), along with the RNA helicase RhlB and the glycolytic enzyme enolase, to form the core RNA degradosome. PNPase can also bind to the RNA chaperone Hfq (Mohanty et al. 2004). All of the pnp mutants we obtained had mutations that led to amino acid substitutions in the N-terminal domain of PNPase, and all of the pnp mutants were cold-sensitive, indicating that these were loss-of-function mutations (Fig. 2). This domain contains the two RNase $\mathrm{PH}$ domains that are involved in catalysis and in binding to RNase E (Duran-Figueroa et al. 2006).

Unexpectedly for a mutation that inactivates an exonuclease, deletion of $p n p$ decreased the half-life of the RyhB, SgrS, and CyaR sRNAs (Fig. 3) and also led to a decrease in the steady-state levels of the $p t s G$, $\operatorname{sod} B$, and ompX encoding mRNAs (Fig. 4). These results are inconsistent with a direct effect of PNPase in initiating degradation of these sRNAs and mRNAs, in which case we might have expected increased levels of these RNAs and increased half-lives.

Instead, our data suggest that $p n p$ mutants failed to carry out sRNA regulation because the sRNAs were degraded before they were able to pair with their targets. Prior to this work, Hfq was the only protein known to protect sRNAs from degradation by RNase E. However, a pnp deletion mutant had the same level of $\mathrm{Hfq}$ as the wild-type strain (Fig. 5A), ruling out a model in which PNPase regulates Hfq amounts. An alternative model was that PNPase is necessary for Hfq activity, assisting $\mathrm{Hfq}$ in binding sRNAs either directly or by displacing already bound sRNAs from Hfq. In this model, the sRNAs were rapidly degraded because they were not binding to Hfq. However, we saw additive effects of $p n p$ and $h f q$ mutants in the degradation of RyhB and SgrS (Fig. 3A,B), strongly suggesting that the effect of PNPase was not fully dependent upon Hfq. In addition, in vitro studies do not support a requirement for PNPase for Hfq to bind sRNAs (Mikulecky et al. 2004; Fender et al. 2010). If PNPase was required to displace already bound sRNAs, then we would have expected that inducing new synthesis of Hfq just prior to inducing the expression of an sRNA would have suppressed the observed defect in sRNA regulation in an pnp mutant. This was not the case (Fig. 5B,C), and in vitro studies have shown that 


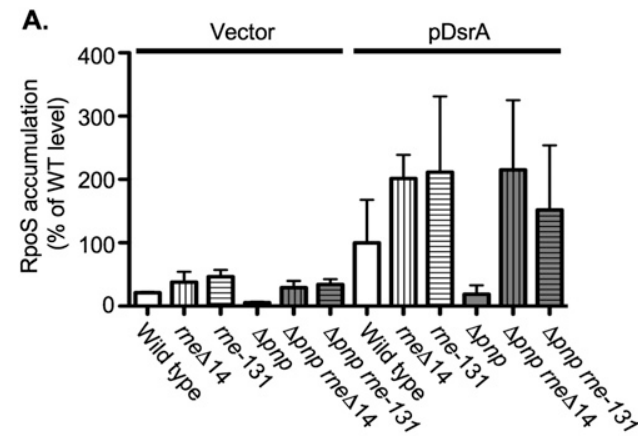

B.

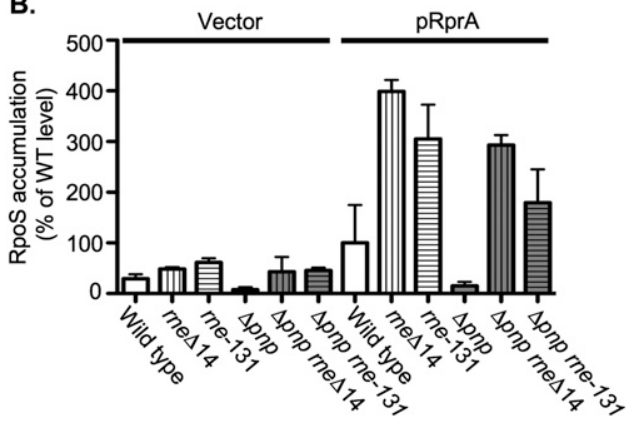

FIGURE 9. Analysis of the effect of a $p n p$ deletion and the rne $\Delta 14$ and rne-131 alleles on positive regulation of RpoS expression by DsrA and RprA. Western blot analysis of RpoS from exponential phase cultures of isogenic $p n p^{+}$and $\Delta p n p$ strains either wild-type for rne (DJ624 and NRD579) or carrying rne 144 (NRD475 and NRD589) or rne-131 (NRD476 and NRD578) harboring either a vector or a plasmid that expresses DsrA $(A)$ or RprA $(B)$ from a lac promoter. Overnight cultures were diluted 200-fold in fresh LB medium with ampicillin, growth at $37^{\circ} \mathrm{C}$ to an $\mathrm{OD}_{600}$ of $0.3-0.4$, the sRNA was induced by addition of IPTG for $20 \mathrm{~min}$. Samples were then taken, the protein TCA-precipitated, and an equal amount of protein based on the cell density of each culture processed as described in Materials and Methods and developed using an anti-RpoS polyclonal antibody. The band intensity from each lane was quantified using the Multi Gauge software. The band intensity for the wild-type strain was set to $100 \%$, and other samples were normalized to the wild-type strain. These results represent the mean of two experiments.

sRNAs can rapidly displace one another from purified Hfq in the absence of additional proteins by a concentrationmediated effect (Fender et al. 2010). Therefore, we conclude that the defect in sRNA regulation in a $p n p$ deletion strain was not via lowering the expression level of Hfq or by reducing the ability of $\mathrm{Hfq}$ to bind sRNAs.

A model that is consistent with our data is that PNPase protects $s R N A s$ prior to pairing by limiting access of RNase $\mathrm{E}$ to Hfq-bound sRNAs. The $\mathrm{C}$ terminus of RNase E, a scaffold for binding of RhlB, enolase, and PNPase, also interacts with Hfq. The site of interaction of Hfq appears to overlap with the RhlB/enolase binding site, present in $r n e \Delta 10$ but deleted in $r n e-131$ and $r n e \Delta 14$ (Morita et al. 2005). In this model, the loss-of- function mutations in $p n p$ allow Hfq-bound sRNAs to be accessed by the RNase E prematurely, leading to the inappropriate rapid degradation of these sRNAs. However, in the rne-131 or the rne $\Delta 14$ mutant, the interaction of RNase $\mathrm{E}$ with the Hfq-bound
sRNA is reduced, whether or not PNPase is present, due to the loss of the RNase $\mathrm{E}$ domain involved in the normal interaction. As a result, there is reduced degradation of the sRNA in the presence or absence of PNPase. In addition, the C-terminal deletion in RNase $\mathrm{E}$ may also contribute to the suppression by reducing the general degradation activity of RNase E. PNPase may also be decreasing the rate of degradation of sRNAs by negatively regulating RNase E activity through allosteric effects achieved from binding to the C-terminal scaffold domain, or by blocking the binding of other proteins to the C-terminal scaffold domain that may enhance its activity. This may explain the additive effects of the $h f q$ and $p n p$ deletion in increasing the degradation of sRNAs (Fig. 3A,B). Consistent with a general regulation of the catalytic activity of RNase E by the C-terminal scaffold domain, previous studies by Leroy et al. (2002) demonstrated that deletion of the whole $\mathrm{C}$ terminus (rne131) significantly reduced the ability of RNase E to degrade a model untranslated substrate in vivo, while deletion of just the PNPase binding site (rne $\Delta 10)$ increased activity. In another study, a deletion similar to $r n e \Delta 10$, rne-225, increased the turnover of $l p p$, while longer deletions reversed this effect (Ow et al. 2000).

Why does truncation of the C-terminal scaffold domain that includes the RhlB and enolase binding site of RNase E suppress the cold sensitivity of the PNPase mutant? PNPase is up-regulated at low temperatures (Mathy et al. 2001). The assumption has been that PNPase directly participates in the degradation of structured mRNAs at low temperature. In the absence of PNPase, the turnover of mRNAs encoding several cold-shock proteins is decreased, and the stabilization of these mRNAs, which allows continued expression of cold-shock proteins, presumably causes the growth arrest of $p n p$ mutants at low temperatures (Yamanaka and Inouye 2001). However, our finding that rne truncations suppressed cold sensitivity suggests that the loss of PNPase is harmful at low temperature due instead to the resulting overdegradation or unregulated degradation of some sRNAs and mRNAs.

There have been previous reports on the effect of a $p n p$ deletion on the turnover of sRNAs, with different results from the result here. In our previous work (Massé et al. 2003), we found that a pnp mutant did not affect RyhBdependent regulation of $\operatorname{sodB}$. We retested the pnp::Tn5 strain used, EM1368, and found that it did not show cold sensitivity and that the kanamycin resistance marker was not linked to the pnp region (data not shown). Therefore, it seems likely that the Tn5 transposon moved elsewhere during construction of this strain. In the current work, we observed significant growth defects for $p n p$ mutants under all conditions, highlighting some of the difficulties of working with these strains and the possibility of selecting suppressors. Whether the other differences with published results reflect different roles for PNPase for different sRNAs or are due to different strains and different growth conditions is not yet 


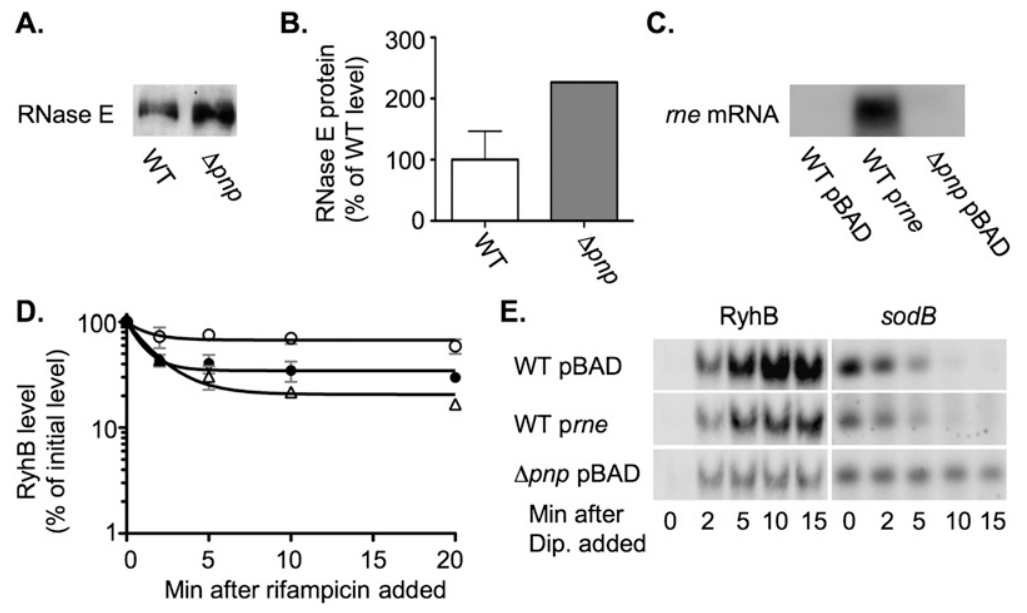

FIGURE 10. RNase E levels in pnp mutants and the effect of overexpression of RNase E on sRNA function. $(A, B)$ Overnight cultures of the wild-type strain TM338 that expresses a FLAG-tagged RNase E from its native promoter (WT; white bars) or the isogenic pnp deletion mutant NRD558 ( $\Delta p n p$; gray bars) were diluted 200-fold into fresh LB. The strains were grown at $37^{\circ} \mathrm{C}$ to an $\mathrm{OD}_{600}$ between 0.3 and 0.4 , and a sample was removed from each culture and TCA-precipitated. An equal amount of protein based on the cell density was processed as described in Materials and Methods and developed using an anti-FLAG monoclonal affinity-purified antibody. A representative blot is shown in $A$. The results shown in $B$ represent the mean of two independent experiments. $(C, D, E)$ Overnight cultures of the wild-type strain (NM534; WT; circles) or a derivative harboring a pnp deletion (NRD571; $\Delta p n p$; triangles) and carrying the empty vector (pBAD30; pBAD; open symbols) or a plasmid that expresses RNase E from an araBAD promoter (pNRD416; prne; solid symbols) were diluted 200-fold in LB-ampicillin medium containing arabinose at a final concentration of $0.0009 \%$. The strains were grown at $37^{\circ} \mathrm{C}$ to an $\mathrm{OD}_{600}$ between 0.3 and 0.4 , a sample was taken, and dipyridyl was added to each culture. Additional samples were taken after dipyridyl addition as indicated in $E$. Sixteen minutes after dipyridyl addition, rifampicin was added to the cultures, and samples were taken as indicated in $D$. The RNA was extracted and processed as described in Figure 4, using the $5^{\prime}$-biotinylated rne probe, RyhB probe, and sodB probe. The Northern blot in $C$ contains the RNA taken from each sample prior to dipyridyl addition. The blots $C$ and $E$ are representative of two experiments. The results in $D$ represent the mean of two experiments, and the standard deviation is indicated by the gray bars.

known. Viegas et al. found that introduction of a $p n p$ deletion into Salmonella enterica serovar Typhimurium increased the half-life of the MicA and SraL sRNAs threefold during stationary phase (Viegas et al. 2007). In related work, Andrade and Arraiano showed that the half-life of the MicA sRNA is more than three times longer in a $p n p$ deletion mutant than in a wild-type strain of $E$. coli (Andrade and Arraiano 2008). These authors also found that introduction of a pnp deletion into a strain of E. coli substantially decreased the ompA mRNA level, and this decrease was at least partially dependent upon MicA. These results suggest that the increase in the stability of the MicA sRNA in the pnp mutant was responsible for the lower level of the ompA mRNA that was observed. These papers used stationary phase cultures. Andrade and Arraiano found no significant difference in the half-life of the MicA sRNA in a pnp mutant during exponential phase, the condition under which we examined the half-lives of multiple sRNAs.

We note that we did not isolate any mutations in rne itself. The failure to find such mutations probably reflects the conditions of our selection/screening, in which re- pression of translation by the sRNAs was sufficient to give the wild-type phenotype. As confirmation of this, we reconstructed the selection strain with an rne131 mutation. The cirA-lac $Z$ fusion is still repressed by $\mathrm{OmrB}$ in that host (data not shown). Thus, in spite of the clear role of the RNase $\mathrm{E}$ degradosome in degradation of both the mRNA targets and the sRNA, such degradation is not always essential for Hfq-dependent gene regulation (Maki et al. 2008).

Our model leaves a number of questions unanswered. What changes upon pairing, in terms of RNase E access, allowing degradation under these conditions? Possibly pairing to an mRNA directly activates RNase E. Alternatively, pairing leads to Hfq displacement or more efficient competition by other sRNAs for the bound Hfq. Thus, our results suggest that the trafficking of $\mathrm{Hfq}$ on and off RNAs and the interaction with RNase E are more complex than expected. This will be a useful direction for further work.

\section{MATERIALS AND METHODS}

\section{Bacterial strains and plasmids}

All strains used in this study, other than the DY330 strain used for recombineering, are derivatives of E. coli K-12 strain MG1655. Strains and plasmids used in this study are listed in Table 2. The primers and 5'-biotinylated probes used in this study are listed in Table 3 and were supplied by Integrated DNA Technologies, Inc. All transductions were performed using phage P1vir as described by Miller (1992). The yjfP deletion strain NRD336 was generated by $\lambda$ Red recombinase-mediated gene replacement using the PCR product generated from the template plasmid pKD3, using yjfPKO For and yjfPKO Rev primers. Replacement of the entire pnp coding region by a chloramphenicol or kanamycin cassette in strain NRD463 or NRD465, respectively, was accomplished via $\lambda$ Red recombinase-mediated gene replacements using the PCR product generated from the template plasmid $\mathrm{pKD} 3$ or $\mathrm{pKD} 4$, respectively, using the primers pnpKO For and pnpKO Rev.

Plasmid pNRD412 was constructed by amplification of pnp from strain MG1655 using pnp-SacI For and pnp-HindIII Rev primers and subsequently cloning the PCR product into the vector pBAD30 digested with SacI and HindIII. Plasmid pNRD414 was generated by amplification of $h f q$ from strain MG1655 using hfq-EcoRI For and hfq-HindIII Rev primers and then cloning the PCR product into pBAD30 digested with EcoRI and HindIII. Finally, plasmid pNRD416 was made by amplification of $r n e$ from strain MG1655 using primers rne-EcoRI For and rne-XbaI Rev and subsequently cloning the PCR product into pBAD30 digested with EcoRI and XbaI. All of the plasmids were sequenced to verify the fidelity of the cloned gene. 
TABLE 2. Strains and plasmids used in this study

\begin{tabular}{|c|c|c|}
\hline Strain or plasmid & Relevant features & Reference or source \\
\hline \multicolumn{3}{|l|}{ Strains } \\
\hline AZ233 & MC4100 $\Delta h f q::$ cat sacB & G. Storz, NICHHD \\
\hline DJ624 & MG1655 $\Delta /$ acX74 mal::lacl ${ }^{q}$ & D. Jin, $\mathrm{NCl}$ \\
\hline DY330 & W3110 $\lambda$ cl857 $\Delta$ (croA-bio) & (Yu et al. 2000) \\
\hline EM1368 & MG1655 $\Delta / a c X 74$ pnp::Tn5 & (Massé et al. 2003) \\
\hline EM1375 & MG1655 s/acX74 rnes 10 zce-726::Tn 10 & (Massé et al. 2003) \\
\hline EM1376 & MG1655 s/acX74 rnes 14 zce-726::Tn10 & (Massé et al. 2003) \\
\hline EM1377 & MG1655 slacX74 rne-131 zce-726::Tn10 & (Massé et al. 2003) \\
\hline MG1193 & DJ624 $\Delta$ fur::kan $\lambda$ RScirA-lacZ & (Guillier and Gottesman 2008) \\
\hline MG1655 & Wild type & Lab strain collection \\
\hline NM534 & $\Delta a r a 714 \mathrm{P}_{\mathrm{CP} 18} \because: a r a E$ & N. Majdalani, $\mathrm{NCl}$ \\
\hline NRD301 & MG1193 рпрС277T & This study \\
\hline NRD302 & MG1193 pnpC277T & This study \\
\hline NRD303 & MG1193 pnpC277T & This study \\
\hline NRD304 & MG1193 hfqG12A & This study \\
\hline NRD305 & MG1193 pсnBG196A & This study \\
\hline NRD306 & MG1193 pпрC277T & This study \\
\hline NRD307 & MG1193 hfqA23G & This study \\
\hline NRD308 & MG1193 pсnBC733T & This study \\
\hline NRD309 & MG1193 hfqT124C & This study \\
\hline NRD310 & MG1193 hfqG118A & This study \\
\hline NRD311 & MG1193 pсnBC313T & This study \\
\hline NRD312 & MG1193 pпрС277T & This study \\
\hline NRD313 & MG1193 pnpG1307A & This study \\
\hline NRD314 & MG1193 hfqA164G & This study \\
\hline NRD315 & MG1193 hfqA170G & This study \\
\hline NRD316 & MG1193 pnpG1466A & This study \\
\hline NRD317 & MG1193 hfqC46T & This study \\
\hline NRD318 & MG1193 pnpC1310T & This study \\
\hline NRD319 & MG1193 pnpC943T & This study \\
\hline NRD320 & MG1193 pnpG1466A & This study \\
\hline NRD321 & MG1193 pnpG1466A & This study \\
\hline NRD322 & MG1193 pnpG1466A & This study \\
\hline NRD323 & MG1193 hfqA26C & This study \\
\hline NRD324 & MG1193 pсnBG209A & This study \\
\hline NRD325 & MG1193 hfqA170G & This study \\
\hline NRD326 & MG1193 pnpG1466A & This study \\
\hline NRD336 & MG1655 $\Delta y j f P:: c a t$ & This study \\
\hline NRD345 & MG1655 $\Delta$ cyaR::cat & (De Lay and Gottesman 2009) \\
\hline NRD349 & MG1655 $\Delta$ cyaR::kan & This study \\
\hline NRD377 & MG1655 slacX74 mal::lacl ${ }^{q} \mathrm{P}_{\mathrm{BAD}}::$ ompX::lacZ $\Delta c y a R:: c a t$ & (De Lay and Gottesman 2009) \\
\hline NRD428 & MG1193 pcnBG196A (hemL-fhuB)mini-Tn10 cam & NRD305 + P1(NRD429) \\
\hline NRD429 & MG1193 (hemL-fhuB)mini-Tn10 cam & This study \\
\hline NRD430 & MG1193 yhbX::mini-Tn10 cam & This study \\
\hline NRD437 & MG1193 yraN::mini-Tn10 cam & This study \\
\hline NRD441 & MG1193 pnpC277T yhbX::mini-Tn10 cam & NRD303 + P1(NRD430) \\
\hline NRD443 & MG1193 pnpC1310T yraN::mini-Tn10 cam & NRD318 + P1(NRD437) \\
\hline NRD450 & MG1193 pnpG1466A yhbX::mini-Tn10 cam & NRD326 + P1(NRD430) \\
\hline NRD453 & MG1655 $\Delta$ lacX74 mal::lacla pnpC277T yhbX::mini-Tn10 cam & DJ624 + P1(NRD441) \\
\hline NRD457 & MG1655 $\Delta / a c X 74$ mal::laclq pnpC1310T yraN::mini-Tn10 cam & DJ624 + P1(NRD443) \\
\hline NRD459 & MG1655 $\Delta$ lacX74 mal::lacla $\Delta h f q::$ cat sacB & DJ624 + P1(AZ233) \\
\hline NRD461 & MG1193 pnpG1307A yraN::mini-Tn10 cam & NRD313 + P1(NRD437) \\
\hline NRD462 & MG1193 pnpC943T yraN::mini-Tn10 cam & NRD319 + P1(NRD437) \\
\hline NRD463 & MG1655 $\Delta$ pnp::cat & This study \\
\hline NRD465 & MG1655 $\Delta$ pnp::kan & This study \\
\hline NRD473 & MG1655 $\Delta$ lacX74 mal::laclq $\Delta p n p:: k a n$ & DJ624 + P1(NRD465) \\
\hline NRD474 & MG1655 slacX74 mal::laclq rned 10 zce-726::Tn10 & DJ624 + P1(EM1375) \\
\hline NRD475 & MG1655 $\Delta / a c X 74$ mal:: lacl $^{q}$ rnes 14 zce-726::Tn 10 & DJ624 + P1(EM1376) \\
\hline NRD476 & MG1655 slacX74 mal::lacl ${ }^{q}$ rne-131 zce-726::Tn 10 & DJ624 + P1(EM1377) \\
\hline
\end{tabular}


TABLE 2. Continued

\begin{tabular}{|c|c|c|}
\hline Strain or plasmid & Relevant features & Reference or source \\
\hline NRD493 & MG1655 slacX74 mal::lacl ${ }^{q}$ pnp G1466A yhbX::mini-Tn10 cam & DJ624 + P1(NRD450) \\
\hline NRD494 & MG1655 slacX74 mal::laclq pnp G1307A yraN::mini-Tn 10 cam & DJ624 + P1(NRD461) \\
\hline NRD495 & 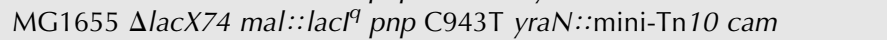 & DJ624 + P1(NRD462) \\
\hline NRD510 & MG1193 pcnB G209A (hemL-fhuB)mini-Tn 10 cam & NRD324 + P1(NRD429) \\
\hline NRD531 & MG1655 $\Delta / a c X 74$ mal::laclq $\Delta c y a R:: k a n$ & DJ624 + P1(NRD349) \\
\hline NRD532 & MG1655 $\Delta$ lacX74 mal::laclq $\Delta$ hfq::cat sacB $\Delta c y a R:: k a n$ & NRD459 + P1(NRD349) \\
\hline NRD533 & 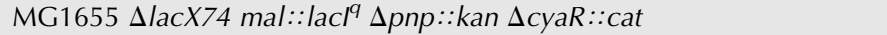 & NRD473 + P1(NRD345) \\
\hline NRD534 & 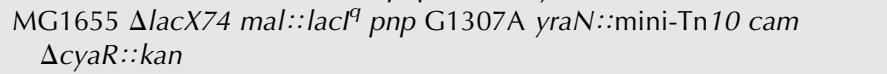 & NRD494 + P1(NRD349) \\
\hline NRD535 & MG1655 $\Delta / a c X 74$ mal::/aclq $\Delta p n p:: k a n \Delta h f q::$ cat sacB & NRD473 + P1(AZ233) \\
\hline NRD540 & MG1655 slacX74 mal::lacl ${ }^{q}$ pcnB G196A (hemL-fhuB)mini-Tn10 cam & DJ624 + P1(NRD428) \\
\hline NRD541 & MG1655 $\Delta / a c X 74$ mal::laclq pcnB G209A (hemL-fhuB)mini-Tn10 cam & DJ624 + P1(NRD510) \\
\hline NRD548 & DJ624 $\Delta$ fur::kan $\lambda$ RScirA-lacZ $\Delta p n p:: c a t$ & MG1193 + P1(NRD463) \\
\hline NRD558 & $\Delta$ pnp::kan rne-FLAG-cat & TM338 + P1(NRD465) \\
\hline NRD571 & $\Delta$ ara714 $\mathrm{P}_{\mathrm{CP} 18}:: a r a E \Delta p n p:: k a n$ & NM534 + P1(NRD465) \\
\hline NRD578 & MG1655 slacX74 mal::lacl ${ }^{q}$ spnp::kan rne-131 zce-726::Tn 10 & NRD473 + P1(EM1377) \\
\hline NRD579 & MG1655 $\Delta / a c X 74$ mal::laclq $\Delta p n p::$ kan zce-726::Tn 10 & NRD473 + P1(EM1377) \\
\hline NRD585 & MG1655 $\Delta / a c X 74$ mal::laclq $\Delta$ pnp::kan rne 110 zce-726::Tn 10 & NRD473 + P1(EM1375) \\
\hline NRD589 & MG1655 $\Delta / a c X 74$ mal::lacl ${ }^{q} \Delta p n p:: k a n$ rne $\Delta 14$ zce-726::Tn 10 & NRD473 + P1(EM1376) \\
\hline NRD672 & $\begin{array}{l}\text { MG1655 } \Delta / a c X 74 \text { mal::lacl }{ }^{q} \mathrm{P}_{\mathrm{BAD}}:: \text { ompX::lacZ } \Delta c y a R:: \text { cat rne } \Delta 10 \\
\text { zce-726::Tn } 10\end{array}$ & NRD377 + P1(EM1375) \\
\hline NRD673 & $\begin{array}{l}\text { MG1655 } \Delta / a c X 74 \text { mal::lacl }{ }^{q} \mathrm{P}_{\mathrm{BAD}}:: \text { ompX::lacZ } \Delta c y a R:: c a t \text { rne } \Delta 14 \\
\text { zce-726::Tn10 }\end{array}$ & NRD377 + P1(EM1376) \\
\hline NRD674 & $\begin{array}{l}\text { MG1655 } \Delta \text { lacX74 mal::lacl }{ }^{q} \mathrm{P}_{\mathrm{BAD}}:: \text { ompX:: lacZ } \Delta \text { cyaR::cat rne-131 } \\
\text { zce-726::Tn10 }\end{array}$ & NRD377 + P1(EM1377) \\
\hline NRD677 & MG1655 $\Delta / a c X 74$ mal::lacla $\mathrm{P}_{\mathrm{BAD}}::$ ompX::lacZ $\Delta c y a R::$ cat $\Delta p n p:: k a n$ & NRD377 + P1(NRD465) \\
\hline NRD678 & 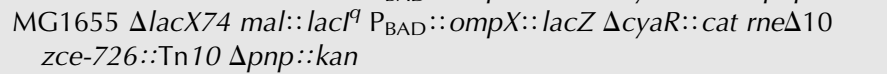 & NRD672 + P1(NRD465) \\
\hline NRD679 & $\begin{array}{l}\text { MG1655 } \Delta \text { lacX74 mal::laclq } \mathrm{P}_{\mathrm{BAD}}:: \text { ompX::lacZ } \Delta c y a R:: \text { cat rne } \Delta 14 \\
\text { zce-726::Tn10 } \Delta \text { pnp::kan }\end{array}$ & NRD673 + P1(NRD465) \\
\hline NRD680 & $\begin{array}{l}\text { MG1655 } \Delta / a c X 74 \text { mal:: lacl }{ }^{q} \mathrm{P}_{\mathrm{BAD}}:: \text { ompX::lacZ } \Delta c y a R:: c a t \text { rne-131 } \\
\text { zce-726::Tn10 } \Delta \text { pnp::kan }\end{array}$ & NRD674 + P1(NRD465) \\
\hline TM338 & rne-FLAG-cat & (Morita et al. 2004) \\
\hline \multicolumn{3}{|l|}{ Plasmids } \\
\hline pKD3 & $\mathrm{Amp}^{\mathrm{r}} \mathrm{Cm}^{\mathrm{r}}$; oriR6Ky; cat cassette flanked by FRT sites & (Datsenko and Wanner 2000) \\
\hline pKD4 & $\mathrm{Amp}^{r} \mathrm{Kan}^{\mathrm{r}}$; oriR6Ky; kan cassette flanked by FRT sites & (Datsenko and Wanner 2000) \\
\hline pKD46 & $\begin{array}{l}\text { Ampr; RepA101(Ts); } \lambda \text { exo, } \gamma \text {, and } \beta \text { expressed from an araBAD } \\
\text { promoter }\end{array}$ & (Datsenko and Wanner 2000) \\
\hline pBAD30 & Ampr; pACYC184 ori; araBAD promoter-based expression vector & (Guzman et al. 1995) \\
\hline pBR-plac & $\begin{array}{l}\text { Ampr; lac promoter-based vector expression vector having a pBR322 } \\
\text { origin. }\end{array}$ & (Guillier and Gottesman 2006) \\
\hline pBR-plac-OmrB & Ampr; expresses OmrB from a lac promoter & (Guillier and Gottesman 2006) \\
\hline pDsra & Ampr; expresses DsrA from a lac promoter & (Mandin and Gottesman 2009) \\
\hline pNK2884 & $\begin{array}{l}\text { Amp }^{r}, \mathrm{Cm}^{r} ; \mathrm{pMB} 1 \text { ori; mini-Tn } 10 \text { cam and transposase expressed from a } \\
\mathrm{P}_{\text {tac }} \text { promoter }\end{array}$ & (Kleckner et al. 1991) \\
\hline pNRD405 & Ampr; expresses CyaR from a lac promoter & (De Lay and Gottesman 2009) \\
\hline pNRD412 & $\begin{array}{l}\text { Sacl-HindIII pnp containing fragment cloned into the same sites in } \\
\text { pBAD30 }\end{array}$ & This study \\
\hline pNRD414 & $\begin{array}{l}\text { EcoRI-HindlII } h f q \text { containing fragment cloned into the same sites in } \\
\text { pBAD30 }\end{array}$ & This study \\
\hline pNRD416 & $\begin{array}{l}\text { Xbal-EcoRI rne containing fragment cloned into the same sites in } \\
\text { pBAD30 }\end{array}$ & This study \\
\hline pRprA & Amp r; expresses RprA from a lac promoter & (Mandin and Gottesman 2009) \\
\hline
\end{tabular}

\section{Culture media and growth conditions}

Strains were grown in liquid medium or agar plates containing Lennox broth or M63 supplemented with vitamin B1 at a final concentration of $0.001 \%$ and glucose or succinate at a final concentration of $0.2 \%$. When medium was supplemented with arabinose, arabinose was used at a final concentration of $0.1 \%$, unless otherwise specified. Antibiotics were used at the following concentrations: ampicillin, $100 \mathrm{mg} \mathrm{L}^{-1}$; chloramphenicol, $10 \mathrm{mg}$ $\mathrm{L}^{-1}$; kanamycin, $25 \mathrm{mg} \mathrm{L}^{-1}$; rifampicin, $250 \mathrm{mg} \mathrm{L}{ }^{-1}$; and 
TABLE 3. Primers and probes used in this study

\begin{tabular}{|c|c|}
\hline Primer or probe & Sequence $\left(5^{\prime}-3^{\prime}\right)$ \\
\hline \multicolumn{2}{|l|}{ Primers } \\
\hline AD1 & NTCGASTWTSGWGTT \\
\hline AD2 & NGTCGASWGANAWGAA \\
\hline AD3 & WGTGNAGWANCANAGA \\
\hline hfq For & GCAGATAACCTGGCTGCG \\
\hline hfg Rev & GCTCACCAGCATCATAACG \\
\hline hfq-EcoRI For & GGGAATTCATATAAGGAAAAGAGAGAATGGC \\
\hline hfq-HindIII Rev & GGAAGCTTCCTTATTCGGTTTCTTCGCTG \\
\hline pnpKO For & $\begin{array}{l}\text { ACCAGTGCCGTAAGGTACTGTCTAAGAAAGAGAAAGGATAT } \\
\text { TACAGTGTAGGCTGGAGCTGCTTC }\end{array}$ \\
\hline pnpKO Rev & $\begin{array}{l}\text { GCCCGGTTAAAAGCCCCCCGCCGCAGCGGAGGGCAAATG } \\
\text { GCAACССАTATGAАTATССТССТTAG }\end{array}$ \\
\hline pnp-Sacl For & GAGCTCCAGTGCCGTAAGGTACTG \\
\hline pnp-HindIII Rev & AAGCTTGGCAAATGGCAACСТTACTCG \\
\hline rne-EcoRI For & GGGAATTCGAGTAAGTTACGATGAAAAGAATG \\
\hline rne-Xbal Rev & GGTCTAGAGCAAGGATGCCATTCGATG \\
\hline Tn10cam487 & GAGGTGCTCСАGTGGСTTC \\
\hline Tn10cam580 & ССТССТGTTCAGСТАСТGACG \\
\hline Tn10cam3500 & ССАGTTTGСТСАGGСТСТСС \\
\hline Tn10cam3616 & ССGTGTGСТTCTCAAATGCC \\
\hline yjfPKO For & $\begin{array}{l}\text { TAAGGATTTCTTCATGTCATACCAGACAAAAAGGAGAGACAG } \\
\text { ATGGTGTAGGCTGGAGCTGCTTC }\end{array}$ \\
\hline yjfPKO Rev & $\begin{array}{l}\text { GCAAGGGGTCAGCATTCTGCGTGTTTAAAGATGCTGGCGG } \\
\text { AAAAACATATGAATATCCTCCTTAG }\end{array}$ \\
\hline \multicolumn{2}{|l|}{ Probes } \\
\hline CyaR probe & TGGTTCCTGGTACAGCTAGCATTTTATGGGTTATG \\
\hline lacZ probe & GGCCGTAACCGACCCAGCGCCCGTTGCACCACAGA \\
\hline ompX probe & TACGGAAGTACCTGCGGTGAAAGCCAGAACTGCGG \\
\hline ptsG probe & CAGCCAGCTGAAATTCGCGGAACCGACGCCCAGCAG \\
\hline rne probe & CGCGCACGCTACGGTCAACGGCATTGGCGACTGGG \\
\hline RyhB probe & $\begin{array}{l}\text { AAGTAATACTGGAAGCAATGTGAGCAATGTCGTGCTTTCAG } \\
\text { GTTCTC }\end{array}$ \\
\hline SgrS probe & GCAACCAGCACAACTTCGCTGTCGCGGTAAAATAGTG \\
\hline sodB probe & TGCGATAGTCGATGTAATAAGCGTGTTCCCAGACATCAACC \\
\hline ssrA probe & 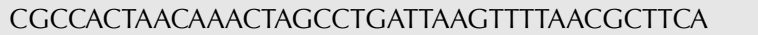 \\
\hline
\end{tabular}

than 100 -fold, to better than $1 / 10^{7}$. A total of 26 mutants from three independent pools of mutagenesis and one spontaneous mutant that survived the selection and passed the screen were further characterized.

\section{Generation of a pool of random transposants}

Strain MG1193 was transformed with plasmid pNK2884. An overnight culture of strain MG1193 harboring pNK2884 was diluted 200-fold into fresh LB medium containing ampicillin and grown at $37^{\circ} \mathrm{C}$ to an $\mathrm{OD}_{600}$ of 0.3-0.4. Transposition of the miniTn10 cam was induced for $1 \mathrm{~h}$ with IPTG, and the cells were then pelleted at $4500 \mathrm{rpm}$ for $10 \mathrm{~min}$. The supernatant was removed, and the cells were suspended in fresh LB to a final $\mathrm{OD}_{600}$ between 0.1 and 0.2. Phage P1vir was then added to the culture, and a P1 lysate was generated as described by Miller (1992).

\section{Mapping of mutations}

Preliminary experiments demonstrated that loss of $h f q$ activity would allow cells to pass the selection/screening procedure. Thus, the initial step in mapping was to identify any $h f q$ mutants that were obtained. All mutants, originally selected for growth on succinate, were transduced with a P1 lysate grown on strain NRD336 containing yjfP::cat, a transposon linked to $h \mathrm{fq}^{+}$, and transductants were selected on M63 agar plates containing vitamin B1, glucose, and chloramphenicol at $37^{\circ} \mathrm{C}$. The chloramphenicol-resistant trans-

tetracycline, $25 \mathrm{mg} \mathrm{L}^{-1}$. 2,2'-Dipyridyl (dipyridyl) or $\alpha$-methyl$\mathrm{D}$-glucoside $(\alpha-\mathrm{MG})$ was added to Lennox broth liquid medium or agar plates at a final concentration of $250 \mu \mathrm{M}$ or $0.5 \%$, respectively. XG and IPTG were used at a final concentration of $20 \mathrm{mg} \mathrm{L}^{-1}$ and $100 \mu \mathrm{M}$, respectively.

\section{Mutagenesis}

Chemical mutagenesis of strain MG1193 harboring plasmid pBRplac-OmrB was performed using 2-aminopurine as described by Miller (1992). Briefly, $50 \mu \mathrm{L}$ of a $10^{-4}$ dilution of an overnight culture of strain MG1193 harboring pBr-plac-OmrB was added to three tubes containing $3.0 \mathrm{~mL}$ of $\mathrm{LB}$ liquid medium with ampicillin and 2-aminopurine $\left(700 \mathrm{mg} \mathrm{L}^{-1}\right)$. After $24 \mathrm{~h}$ incubation at $37^{\circ} \mathrm{C}$, the cultures were serially diluted 10 -fold, and the dilutions were plated on minimal M63 agar plates containing ampicillin, vitamin $\mathrm{B} 1$, and succinate and incubated at $37^{\circ} \mathrm{C}$. Colonies that formed on the succinate minimal plates were restreaked onto glucose minimal plates containing ampicillin, IPTG, and X-Gal. In the absence of mutagenesis, mutations with the desired phenotype (growth on succinate, blue on X-Gal) arose at around $1 / 10^{9}$. After mutagenesis, the rate was increased more ductants were then tested for growth on succinate by restreaking onto M63 agar plates with vitamin B1 and either glucose or succinate and incubated at $37^{\circ} \mathrm{C}$. For those mutants that produced a significant number of chloramphenicol-resistant transductants that no longer grew on succinate minimal plates, suggesting the presence of an $h f q$ mutation enabling succinate growth in the original mutant strain, the $h f q$ allele from the original mutant was amplified and sequenced using the primers hfq For and hfq Rev.

Those mutants that yielded only chloramphenicol-resistant transductants that had retained the ability to grow on minimal succinate media were concluded to be somewhere other than $h f q$ and were treated as follows to identify a transposon linked to the mutation (Kleckner et al. 1991). The mutants were transduced with a P1vir lysate grown on a pool of mini-Tn10 cam transposants generated in the wild-type parental strain MG1193. Transductants were plated on MacConkey-lactose agar plates with ampicillin and chloramphenicol and incubated at $37^{\circ} \mathrm{C}$ and the rare (1/200) $\mathrm{Lac}^{-}$transductants identified; in these strains, the negative regulation of the cirA-lac $Z$ fusion by $\mathrm{OmrB}$ is restored. Thus, the transposon insertion in these transductants should be cotransducible with the wild-type allele of the original unmapped mutation. The cotransduction frequency of the transposon and 
the $\mathrm{Lac}^{-}$(wild type) phenotype was determined by transducing the original mutant again, this time with a P1vir lysate grown on a $\mathrm{Lac}^{-}$transductant, and selecting for the acquisition of the transposon and screening for the loss of the $\mathrm{Lac}^{+}$(mutant) phenotype on MacConkey-lactose agar plates with ampicillin and chloramphenicol. Those transductants that acquired the $\mathrm{Lac}^{-}$phenotype were also tested for the ability to grow on minimal plates containing succinate as the sole carbon source to assess whether the ability of RyhB to negatively regulate the $s d h C D A B$ mRNA was also restored.

The location of the transposon linked to the $\mathrm{Lac}^{-}$phenotype was identified by thermal asymmetric interlaced (TAIL) PCR from 200 ng of purified genomic DNA, according to Liu et al., using primer Tn10cam487 along with primer AD1, AD2, or AD3 (Liu et al. 1995). The resulting PCR product was diluted 40-fold in ultrapure water and was subsequently used as a template for a second PCR reaction using the primers Tn10cam580 and AD1, $\mathrm{AD} 2$, or $\mathrm{AD} 3$. The product of this subsequent PCR was then purified using a Qiagen MinElute PCR purification kit and then sequenced using the Tn10cam580 primer. After the insertion site of the transposon was identified by sequencing, sequencing was then performed on the most promising candidate genes in the vicinity of the transposon to identify the mutation responsible for the $\mathrm{Lac}^{+}$phenotype in the mutant. Three of these transposon insertions, present in NRD429, NRD430, and NRD437, were used in further analysis.

\section{Northern blotting}

The effects of $h f q$, pnp, and rne mutations on levels and turnover of sRNAs and mRNAs were tested by Northern blotting. For the determination of RyhB and $\operatorname{sodB}$ turnover, overnight cultures were diluted 200-fold into fresh LB medium and incubated at $37^{\circ} \mathrm{C}$. A $700-\mu \mathrm{l}$ sample was removed from each culture when an $\mathrm{OD}_{600}$ between 0.3 and 0.4 was reached, and dipyridyl was added to each culture. Additional samples were removed 2, 5, 10, and $15 \mathrm{~min}$ after dipyridyl addition. Sixteen minutes after dipyridyl addition, each culture was treated with rifampicin, and additional samples were removed at appropriate intervals after treatment. RNA was extracted from each sample by the hot phenol method previously described by Massé et al. (2005). To examine the effect of overexpression of Hfq on the turnover of RyhB and the $\operatorname{sodB}$ mRNA, overnight cultures of strains harboring pBAD30 or pNRD414 (pBAD30- $h f q^{+}$) were diluted 200-fold into fresh LB medium containing ampicillin. When cultures reached an $\mathrm{OD}_{600}$ between 0.3 and 0.4 , arabinose was added to each culture to a final concentration of $0.2 \%$. A $700-\mu \mathrm{L}$ sample was removed from each culture $5 \mathrm{~min}$ after arabinose addition, and dipyridyl was then added to each culture. Additional samples were removed 2, 5, 10, and $15 \mathrm{~min}$ after dipyridyl addition. Rifampicin was added to each culture $16 \mathrm{~min}$ after dipyridyl addition, and samples were removed and RNA extracted as described above.

To examine the turnover of SgrS and $p t s G$ mRNA, overnight cultures were diluted 200-fold into fresh LB medium and incubated at $37^{\circ} \mathrm{C}$. A $700-\mu \mathrm{L}$ sample was removed from each culture when an $\mathrm{OD}_{600}$ between 0.3 and 0.4 was reached, and $\alpha-\mathrm{MG}$ was added to each culture. Additional samples were removed 2, 5, 10, and 15 min after $\alpha$-MG addition. Sixteen minutes after $\alpha-M G$ was added, rifampicin was added to each culture, and samples were removed 2, 5, 10, and $20 \mathrm{~min}$ after rifampicin treatment. RNA was extracted from each sample as described above. To determine the turnover of CyaR and the ompX and ompX' $X^{\prime}$ lacZ mRNA, overnight cultures were diluted 200-fold into fresh LB medium and incubated at $37^{\circ} \mathrm{C}$. When the $\mathrm{OD}_{600}$ of each culture was between 0.3 and 0.4 , a $700-\mu L$ sample was taken, and then IPTG was added to each culture. Additional samples were taken from each culture 2, 5, 10, and 15 min after IPTG addition, and 16 min after IPTG addition, rifampicin was added to each culture. Samples were removed after rifampicin treatment, and RNA was extracted as described above for RyhB and SgrS.

Northern blot analysis of RyhB, SgrS, and CyaR expression and turnover was performed by fractionating $3 \mu \mathrm{g}$ of RNA from each sample on a Bio-Rad Criterion 10\% Tris-borate-EDTA (TBE)-urea polyacrylamide gel in $1 \times \mathrm{TBE}$ at $55 \mathrm{~V}$ for $3 \mathrm{~h}$ after prerunning the gel at $55 \mathrm{~V}$ for $30 \mathrm{~min}$. The fractionated RNA was then transferred by electroblotting to a Zeta-Probe GT membrane (Bio-Rad) at $200 \mathrm{~mA}$ for $2 \mathrm{~h}$ in $0.5 \times$ TBE. The RNA was cross-linked to the membrane by UV irradiation, and the membrane was then probed with the appropriate sRNA probe in ULTRAhyb solution (Ambion) at $42^{\circ} \mathrm{C}$ overnight. The blot was then developed using the Brightstar Biodetect kit (Ambion) as per the manufacture's instructions.

Northern blot analysis of mRNA turnover or rne expression level was performed by fractionating $10 \mu \mathrm{g}$ of RNA from each sample on a $1.2 \%$ agarose gel that was prerun for $5 \mathrm{~min}$ at 12 $\mathrm{V} / \mathrm{cm}$ in a $1 \times$ morpholinepropanesulfonic acid (MOPS) buffer and subsequently run at $5 \mathrm{~V} / \mathrm{cm}$ for $2 \mathrm{~h}$. The RNA was transferred to a Zeta-Probe GT membrane (Bio-Rad) by capillary action overnight, and cross-linked to the membrane by UV irradiation. The membrane was probed with the appropriate mRNA probe in ULTRAhyb solution (Ambion) at $42^{\circ} \mathrm{C}$ overnight and then developed as described above. For a given experiment, all of the RNA samples fractionated were loaded with an equivalent amount of RNA, run on the same agarose gel, transferred to the same membrane, and developed for the same amount of time to allow direct comparisons between different genetic backgrounds.

\section{$\boldsymbol{\beta}$-galactosidase assays}

To determine the expression of the omp $X^{\prime}-{ }^{\prime} l a c Z$ translational fusion, overnight cultures of strains harboring the vector $\mathrm{pBr}$-plac or pNRD405 were diluted 200-fold into fresh LB medium containing ampicillin and IPTG and grown to an $\mathrm{OD}_{600}$ between 0.3 and 0.4. Samples were then removed from each culture, and a $\beta$-galactosidase assay was performed as described by Miller (1992).

\section{Western blotting}

To determine the expression level of Hfq or RNase E, overnight cultures of the relevant strains were diluted 200-fold into fresh LB medium and incubated at $37^{\circ} \mathrm{C}$. A $1.0-\mathrm{mL}$ sample was removed from each culture when the $\mathrm{OD}_{600}$ reached 1 (for $\mathrm{Hfq}$ ) or between 0.3 and 0.4 (for RNase E). For the RpoS Western blots, overnight cultures of each strain were diluted 200 -fold into fresh LB medium with ampicillin and incubated at $37^{\circ} \mathrm{C}$. When cells were between 0.3 and 0.4 , IPTG was added for $20 \mathrm{~min}$ to induce the sRNAs and a $1.0-\mathrm{mL}$ sample was removed. All samples were immediately added to a tube containing $110 \mu \mathrm{L}$ of an ice-cold $50 \%$ solution of trichloroacetic acid (TCA). The sample was incubated on ice for $10 \mathrm{~min}$, and subsequently the precipitated 
protein was pelleted by centrifugation at 13,000 rpm for $10 \mathrm{~min}$. The supernatant was removed, and the pellet was suspended in an $80 \%$ acetone solution. The washed protein was pelleted by centrifugation, the supernatant was removed, and the sample was allowed to air dry for $10 \mathrm{~min}$. The protein was then suspended in $1 \times$ SDS sample buffer (New England Biolabs).

Western blot analysis of $\mathrm{Hfq}$ was performed by fractionating the collected protein on a NuPAGE Novex 12\% Bis-Tris polyacrylamide gel (Invitrogen) in NuPAGE MES SDS running buffer (Invitrogen) at $180 \mathrm{~V}$. The fractionated protein was then transferred to a $0.2 \mu \mathrm{m}$-nitrocellulose membrane by electroblotting at $40 \mathrm{~V}$ for $75 \mathrm{~min}$ in NuPAGE transfer buffer (Invitrogen). The membrane was rinsed with $1 \times$ phosphate-buffered saline $(\mathrm{pH} 7.4)$ with $0.1 \%$ TWEEN 20 (PBST), blocked overnight in PBST with $3 \%$ nonfat dry milk and washed 3 times in PBST for $10 \mathrm{~min}$. The membrane was probed with rabbit anti-Hfq antisera diluted 5000-fold in PBST with $0.3 \%$ nonfat dry milk for $1 \mathrm{~h}$ and subsequently, after three additional PBST washes, was probed with alkaline phosphatase-linked anti-rabbit IgG antibody (Cell Signaling Technology) diluted 1000 -fold in PBST with $0.3 \%$ nonfat dry milk for $1 \mathrm{~h}$. After 3 washes with PBST, the membrane was then developed using the Lumi-Phos WB chemiluminescent alkaline phosphatase substrate (Thermo Scientific).

Western blot analysis of RNase E levels was performed by fractionating harvested protein on a NuPAGE $4 \%-12 \%$ Bis-Tris polyacrylamide gel (Invitrogen) in NuPAGE MOPS running buffer at $120 \mathrm{~V}$ for $2 \mathrm{~h}$. The fractionated protein was subsequently transferred to a $0.2-\mu \mathrm{m}$ nitrocellulose membrane by electroblotting and treated as described above, except rabbit anti-FLAG monoclonal antibody (Sigma-Aldrich) was used as the primary antibody at a dilution of 1:1000 in PBST with $0.3 \%$ nonfat dry milk.

Western blot analysis of RpoS was performed by fractionating the collected protein on a NuPAGE Novex $10 \%$ Bis-Tris polyacrylamide gel (Invitrogen) at $180 \mathrm{~V}$ for $1 \mathrm{~h}$ in NuPAGE MOPS SDS running buffer (Invitrogen). The fractionated protein was transferred to a $0.2-\mu \mathrm{m}$ nitrocellulose membrane by electroblotting and treated as described above, except rabbit anti-RpoS antisera was used as the primary antibody at a dilution of 1:5000 in PBST with $0.3 \%$ nonfat dry milk.

\section{ACKNOWLEDGMENTS}

We thank Gisela Storz and members of our laboratory including N. Majdalani, P. Milanesio, D. Schu, K. Moon, A. Battesti, and T. Song for their comments on this manuscript and their advice. We thank H. Aiba and M. Guillier for providing us with strains. This research was supported by the Intramural Research Program of the NIH, National Cancer Institute, Center for Cancer Research.

Received November 8, 2010; accepted March 21, 2011.

\section{REFERENCES}

Andrade JM, Arraiano CM. 2008. PNPase is a key player in the regulation of small RNAs that control the expression of outer membrane proteins. RNA 14: 543-551.

Argaman L, Hershberg R, Vogel J, Bejerano G, Wagner EG, Margalit H, Altuvia S. 2001. Novel small RNA-encoding genes in the intergenic regions of Escherichia coli. Curr Biol 11: 941-950.
Brennan RG, Link TM. 2007. Hfq structure, function, and ligand binding. Curr Opin Microbiol 10: 125-133.

Brown L, Elliott T. 1996. Efficient translation of the RpoS sigma factor in Salmonella typhimurium requires host factor I, an RNA-binding protein encoded by the $h f q$ gene. J Bacteriol 178: 3763-3770.

Carpousis AJ, Van Houwe G, Ehretsmann C, Krisch HM. 1994. Copurification of E. coli RNAase E and PNPase: Evidence for a specific association between two enzymes important in RNA processing and degradation. Cell 76: 889-900.

Chen S, Lesnik EA, Hall TA, Sampath R, Griffey RH, Ecker DJ, Blyn LB. 2002. A bioinformatics based approach to discover small RNA genes in the Escherichia coli genome. Biosystems 65: 157-177.

Datsenko KA, Wanner BL. 2000. One-step inactivation of chromosomal genes in Escherichia coli K-12 using PCR products. Proc Natl Acad Sci 97: 6640-6645.

De Lay N, Gottesman S. 2009. The Crp-activated small noncoding regulatory RNA CyaR (RyeE) links nutritional status to group behavior. J Bacteriol 191: 461-476.

Duran-Figueroa NV, Pina-Escobedo A, Schroeder I, Simons RW, Garcia-Mena J. 2006. Polynucleotide phosphorylase interacts with ribonuclease E through a $\beta \beta \alpha \beta \beta \alpha$ domain. Biochimie 88: 725735.

Fender A, Elf J, Hampel K, Zimmermann B, Wagner EG. 2010. RNAs actively cycle on the Sm-like protein Hfq. Genes Dev 24: 26212626.

Franze de Fernandez MT, Hayward WS, August JT. 1972. Bacterial proteins required for replication of phage Qbeta ribonucleic acid: Purification and properties of host factor I, a ribonucleic acidbinding protein. J Biol Chem 247: 824-831.

Guillier M, Gottesman S. 2006. Remodelling of the Escherichia coli outer membrane by two small regulatory RNAs. Mol Microbiol 59: 231-247.

Guillier M, Gottesman S. 2008. The 5' end of two redundant sRNAs is involved in the regulation of multiple targets, including their own regulator. Nucleic Acids Res 36: 6781-6794.

Guzman LM, Belin D, Carson MJ, Beckwith J. 1995. Tight regulation, modulation, and high-level expression by vectors containing the arabinose PBAD promoter. J Bacteriol 177: 4121-4130.

Jarrige A, Brechemier-Baey D, Mathy N, Duche O, Portier C. 2002. Mutational analysis of polynucleotide phosphorylase from Escherichia coli. J Mol Biol 321: 397-409.

Johansen J, Eriksen M, Kallipolitis B, Valentin-Hansen P. 2008. Down-regulation of outer membrane proteins by noncoding RNAs: Unraveling the cAMP-CRP- and $\sigma^{\mathrm{E}}$-dependent CyaR-ompX regulatory case. J Mol Biol 383: 1-9.

Kajitani M, Ishihama A. 1991. Identification and sequence determination of the host factor gene for bacteriophage Q beta. Nucleic Acids Res 19: 1063-1066.

Kawamoto H, Morita T, Shimizu A, Inada T, Aiba H. 2005. Implication of membrane localization of target mRNA in the action of a small RNA: Mechanism of post-transcriptional regulation of glucose transporter in Escherichia coli. Genes Dev 19: 328338.

Kleckner N, Bender J, Gottesman S. 1991. Uses of transposons with emphasis on Tn10. Methods Enzymol 204: 139-180.

Lease RA, Cusick ME, Belfort M. 1998. Riboregulation in Escherichia coli: DsrA RNA acts by RNA:RNA interactions at multiple loci. Proc Natl Acad Sci 95: 12456-12461.

Leroy A, Vanzo NF, Sousa S, Dreyfus M, Carpousis AJ. 2002. Function in Escherichia coli of the non-catalytic part of RNase E: Role in the degradation of ribosome-free mRNA. Mol Microbiol 45: 12311243.

Liou GG, Chang HY, Lin CS, Lin-Chao S. 2002. DEAD box RhlB RNA helicase physically associates with exoribonuclease PNPase to degrade double-stranded RNA independent of the degradosomeassembling region of RNase E. J Biol Chem 277: 41157-41162.

Liu JD, Parkinson JS. 1989. Genetics and sequence analysis of the $p c n B$ locus, an Escherichia coli gene involved in plasmid copy number control. J Bacteriol 171: 1254-1261. 
Liu YG, Mitsukawa N, Oosumi T, Whittier RF. 1995. Efficient isolation and mapping of Arabidopsis thaliana T-DNA insert junctions by thermal asymmetric interlaced PCR. Plant J 8: 457463.

Lopilato J, Bortner S, Beckwith J. 1986. Mutations in a new chromosomal gene of Escherichia coli $\mathrm{K}-12$, pcnB, reduce plasmid copy number of pBR322 and its derivatives. Mol Gen Genet 205: 285290.

Luttinger A, Hahn J, Dubnau D. 1996. Polynucleotide phosphorylase is necessary for competence development in Bacillus subtilis. Mol Microbiol 19: 343-356.

Majdalani N, Cunning C, Sledjeski D, Elliott T, Gottesman S. 1998. DsrA RNA regulates translation of RpoS message by an antiantisense mechanism, independent of its action as an antisilencer of transcription. Proc Natl Acad Sci 95: 12462-12467.

Majdalani N, Chen S, Murrow J, St John K, Gottesman S. 2001. Regulation of RpoS by a novel small RNA: The characterization of RprA. Mol Microbiol 39: 1382-1394.

Maki K, Uno K, Morita T, Aiba H. 2008. RNA, but not protein partners, is directly responsible for translational silencing by a bacterial Hfa-binding small RNA. Proc Natl Acad Sci 105: 1033210337.

Mandin P, Gottesman S. 2009. A genetic approach for finding small RNAs regulators of genes of interest identifies RybC as regulating the DpiA/DpiB two-component system. Mol Microbiol 72: 551-565.

Mandin P, Gottesman S. 2010. Integrating anaerobic/aerobic sensing and the general stress response through the ArcZ small RNA. EMBO J 29: 3094-3107.

March JB, Colloms MD, Hart-Davis D, Oliver IR, Masters M. 1989. Cloning and characterization of an Escherichia coli gene, pcnB, affecting plasmid copy number. Mol Microbiol 3: 903-910.

Massé E, Gottesman S. 2002. A small RNA regulates the expression of genes involved in iron metabolism in Escherichia coli. Proc Natl Acad Sci 99: 4620-4625.

Massé E, Escorcia FE, Gottesman S. 2003. Coupled degradation of a small regulatory RNA and its mRNA targets in Escherichia coli. Genes Dev 17: 2374-2383.

Massé E, Vanderpool CK, Gottesman S. 2005. Effect of RyhB small RNA on global iron use in Escherichia coli. J Bacteriol 187: 6962-6971.

Mathy N, Jarrige AC, Robert-Le Meur M, Portier C. 2001. Increased expression of Escherichia coli polynucleotide phosphorylase at low temperatures is linked to a decrease in the efficiency of autocontrol. J Bacteriol 183: 3848-3854.

McCullen CA, Benhammou JN, Majdalani N, Gottesman S. 2010. Mechanism of positive regulation by DsrA and RprA sRNAs: Pairing increases translation and protects rpoS mRNA from degradation. J Bacteriol 192: 5559-5571.

Miczak A, Kaberdin VR, Wei CL, Lin-Chao S. 1996. Proteins associated with RNase $\mathrm{E}$ in a multicomponent ribonucleolytic complex. Proc Natl Acad Sci 93: 3865-3869.

Mikulecky PJ, Kaw MK, Brescia CC, Takach JC, Sledjeski DD, Feig AL. 2004. Escherichia coli $\mathrm{Hfq}$ has distinct interaction surfaces for DsrA, rpoS and poly(A) RNAs. Nat Struct Mol Biol 11: 1206-1214.

Miller JH. 1992. A short course in bacterial genetics. Cold Spring Harbor Laboratory Press, Cold Spring Harbor, NY.

Mohanty BK, Maples VF, Kushner SR. 2004. The Sm-like protein Hfq regulates polyadenylation dependent mRNA decay in Escherichia coli. Mol Microbiol 54: 905-920.

Morita T, El-Kazzaz W, Tanaka Y, Inada T, Aiba H. 2003. Accumulation of glucose 6-phosphate or fructose 6-phosphate is responsible for destabilization of glucose transporter mRNA in Escherichia coli. J Biol Chem 278: 15608-15614.
Morita T, Kawamoto H, Mizota T, Inada T, Aiba H. 2004. Enolase in the RNA degradosome plays a crucial role in the rapid decay of glucose transporter mRNA in the response to phosphosugar stress in Escherichia coli. Mol Microbiol 54: 1063-1075.

Morita T, Maki K, Aiba H. 2005. RNase E-based ribonucleoprotein complexes: Mechanical basis of mRNA destabilization mediated by bacterial noncoding RNAs. Genes Dev 19: 2176-2186.

Muffler A, Fischer D, Hengge-Aronis R. 1996. The RNA-binding protein HF-1, known as a host factor for phage QBeta RNA replication, is essential for rpoS translation in Escherichia coli. Genes Dev 10: 1143-1151.

Ow MC, Liu Q, Kushner SR. 2000. Analysis of mRNA decay and rRNA processing in Escherichia coli in the absence of RNase E-based degradosome assembly. Mol Microbiol 38: 854-866.

Papenfort K, Pfeiffer V, Lucchini S, Sonawane A, Hinton JC, Vogel J. 2008. Systematic deletion of Salmonella small RNA genes identifies CyaR a conserved CRP-dependent riboregulator of OmpX synthesis. Mol Microbiol 68: 890-906.

Py B, Causton H, Mudd EA, Higgins CF. 1994. A protein complex mediating mRNA degradation in Escherichia coli. Mol Microbiol 14: 717-729.

Py B, Higgins CF, Krisch HM, Carpousis AJ. 1996. A DEAD-box RNA helicase in the Escherichia coli RNA degradosome. Nature 381: $169-172$.

Sun X, Wartell RM. 2006. Escherichia coli Hfq binds A18 and DsrA domain II with similar 2:1 Hfq6/RNA stoichiometry using different surface sites. Biochemistry 45: 4875-4887.

Valentin-Hansen P, Eriksen M, Udesen C. 2004. The bacterial Sm-like protein Hfq: A key player in RNA transactions. Mol Microbiol 51: $1525-1533$.

Vanderpool CK, Gottesman S. 2004. Involvement of a novel transcriptional activator and small RNA in post-transcriptional regulation of the glucose phosphoenolpyruvate phosphotransferase system. Mol Microbiol 54: 1076-1089.

Vanzo NF, Li YS, Py B, Blum E, Higgins CF, Raynal LC, Krisch HM, Carpousis AJ. 1998. Ribonuclease $\mathrm{E}$ organizes the protein interactions in the Escherichia coli RNA degradosome. Genes Dev 12: 2770-2781.

Viegas SC, Pfeiffer V, Sittka A, Silva IJ, Vogel J, Arraiano CM. 2007. Characterization of the role of ribonucleases in Salmonella small RNA decay. Nucleic Acids Res 35: 7651-7664.

Vogel J, Bartels V, Tang TH, Churakov G, Slagter-Jager JG, Huttenhofer A, Wagner EG. 2003. RNomics in Escherichia coli detects new sRNA species and indicates parallel transcriptional output in bacteria. Nucleic Acids Res 31: 6435-6443.

Wassarman KM, Repoila F, Rosenow C, Storz G, Gottesman S. 2001. Identification of novel small RNAs using comparative genomics and microarrays. Genes Dev 15: 1637-1651.

Waters LS, Storz G. 2008. Regulatory RNAs in bacteria. Cell 136: 615628.

Yamanaka K, Inouye M. 2001. Selective mRNA degradation by polynucleotide phosphorylase in cold shock adaptation in Escherichia coli. J Bacteriol 183: 2808-2816.

Yu D, Ellis HM, Lee EC, Jenkins NA, Copeland NG, Court DL. 2000. An efficient recombination system for chromosome engineering in Escherichia coli. Proc Natl Acad Sci 97: 5978-5983.

Zhang A, Wassarman KM, Rosenow C, Tjaden BC, Storz G, Gottesman S. 2003. Global analysis of small RNA and mRNA targets of Hfq. Mol Microbiol 50: 1111-1124.

Zhou Z, Deutscher MP. 1997. An essential function for the phosphate-dependent exoribonucleases RNase $\mathrm{PH}$ and polynucleotide phosphorylase. J Bacteriol 179: 4391-4395. 

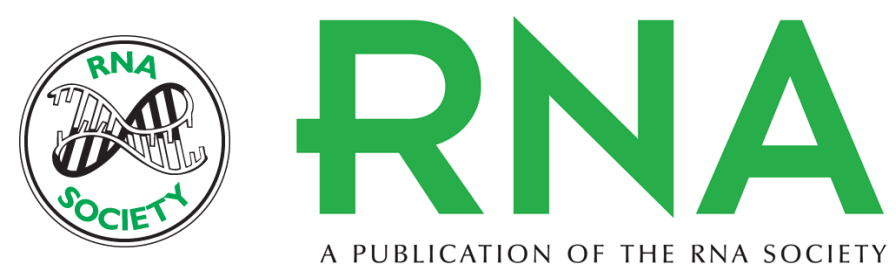

A PUBLICATION OF THE RNA SOCIETY

\section{Role of polynucleotide phosphorylase in sRNA function in Escherichia coli}

Nicholas De Lay and Susan Gottesman

RNA 2011 17: 1172-1189 originally published online April 28, 2011

Access the most recent version at doi:10.1261/rna.2531211

References This article cites 60 articles, 27 of which can be accessed free at:

http://rnajournal.cshlp.org/content/17/6/1172.full.html\#ref-list-1

License

Email Alerting Receive free email alerts when new articles cite this article - sign up in the box at the Service top right corner of the article or click here.

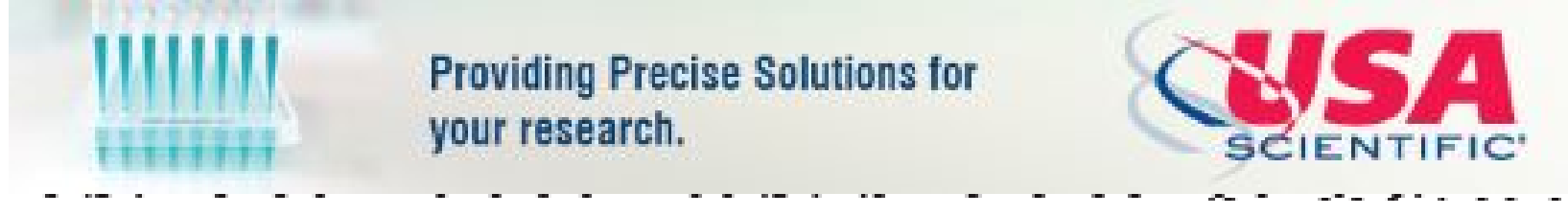

To subscribe to RNA go to:

http://rnajournal.cshlp.org/subscriptions 